\title{
Genomic, transcriptomic, and proteomic approaches towards understanding the molecular mechanisms of salt tolerance in Frankia strains isolated from Casuarina trees
}

Rediet Oshone ${ }^{1}$, Mariama Ngom²,3,4, Feixia Chu' ${ }^{1}$, Samira Mansour ${ }^{5}$, Mame Ourèye Sy ${ }^{2,3}$, Antony Champion ${ }^{2,6}$ and Louis S. Tisa ${ }^{1 *}$

\begin{abstract}
Background: Soil salinization is a worldwide problem that is intensifying because of the effects of climate change. An effective method for the reclamation of salt-affected soils involves initiating plant succession using fast growing, nitrogen fixing actinorhizal trees such as the Casuarina. The salt tolerance of Casuarina is enhanced by the nitrogen-fixing symbiosis that they form with the actinobacterium Frankia. Identification and molecular characterization of salt-tolerant Casuarina species and associated Frankia is imperative for the successful utilization of Casuarina trees in saline soil reclamation efforts. In this study, salt-tolerant and salt-sensitive Casuarina associated Frankia strains were identified and comparative genomics, transcriptome profiling, and proteomics were employed to elucidate the molecular mechanisms of salt and osmotic stress tolerance.

Results: Salt-tolerant Frankia strains (Ccl6 and Allo2) that could withstand up to $1000 \mathrm{mM} \mathrm{NaCl}$ and a salt-sensitive Frankia strain (CCl3) which could withstand only up to $475 \mathrm{mM} \mathrm{NaCl}$ were identified. The remaining isolates had intermediate levels of salt tolerance with MIC values ranging from $650 \mathrm{mM}$ to $750 \mathrm{mM}$. Comparative genomic analysis showed that all of the Frankia isolates from Casuarina belonged to the same species (Frankia casuarinae). Pangenome analysis revealed a high abundance of singletons among all Casuarina isolates. The two salt-tolerant strains contained 153 shared single copy genes (most of which code for hypothetical proteins) that were not found in the salt-sensitive(CCl3) and moderately salttolerant (CeD) strains. RNA-seq analysis of one of the two salt-tolerant strains (Frankia sp. strain Ccl6) revealed hundreds of genes differentially expressed under salt and/or osmotic stress. Among the 153 genes, 7 and 7 were responsive to salt and osmotic stress, respectively. Proteomic profiling confirmed the transcriptome results and identified 19 and 8 salt and/ or osmotic stress-responsive proteins in the salt-tolerant $(\mathrm{Ccl})$ and the salt-sensitive (Ccl3) strains, respectively.

Conclusion: Genetic differences between salt-tolerant and salt-sensitive Frankia strains isolated from Casuarina were identified. Transcriptome and proteome profiling of a salt-tolerant strain was used to determine molecular differences correlated with differential salt-tolerance and several candidate genes were identified. Mechanisms involving transcriptional and translational regulation, cell envelop remodeling, and previously uncharacterized proteins appear to be important for salt tolerance. Physiological and mutational analyses will further shed light on the molecular mechanism of salt tolerance in Casuarina associated Frankia isolates.
\end{abstract}

Keywords: Actinobacteria, Actinorhizal symbiosis, Comparative genomics, Salt stress, Salt tolerance, Transcriptomics

\footnotetext{
*Correspondence: louis.tisa@unh.edu

'Department of Molecular, Cellular and Biomedical Sciences, University of

New Hampshire, 46 College Rd, Durham, NH 03824-2617, USA

Full list of author information is available at the end of the article
} 


\section{Background}

Soil salinization is a worldwide problem that is intensifying because of the effects of climate change. Globally, $20 \%$ of total cultivated and 33\% of irrigated agricultural land are affected by high salinity. Salinized areas are expanding at an alarming rate of $10 \%$ per annum due to a variety of factors encompassing low precipitation, high surface evaporation, weathering of native rocks, irrigation with saline water, and poor agricultural practices [1]. By 2050, more than $50 \%$ of the arable lands are predicted to be salinized [2]. Various methods are used to reclaim salt-affected soils. One effective and commonly used method in the reclamation of saltaffected soils involves initiating plant succession using fastgrowing, nitrogen-fixing actinorhizal trees such as the Casuarina [3]. Actinorhizal plants of the genus Casuarina are notable for high salt tolerance [4] and have been used as a green barrier [5-7]. Some Casuarina species are found growing naturally near brackish waters and swamps or in saline soils [8]. In hydroponic medium supplemented with adequate nitrogen, Casuarina glauca, Casuarina obesa, and Casuarina equisetifolia var. incana will withstand up to $500 \mathrm{mM} \mathrm{NaCl}[5]$.

Actinorhizal plants form a nitrogen-fixing symbiosis with the actinobacteria Frankia that results in the formation of root nodule structures where the bacteria are located. The bacteria convert atmospheric dinitrogen into a biologically useful form and supply the plant with nitrogen. Reciprocally, the plant supplies the bacteria with carbon and energy sources. Frankia associate with a broad range of dicotyledonous angiosperms distributed among 24 genera and 8 families $[9,10]$. The symbiosis with Frankia allows actinorhizal host plants to colonize harsh environmental terrains including highly contaminated, dry, poorly-drained, nutrient-poor and salinized soils $[11,12]$.

Like their plant partners, Frankia strains isolated from Casuarina and Allocasuarina are more $\mathrm{NaCl}$ tolerant than other Frankia strains isolated from actinorhizal plant species not normally growing under sodic conditions [13]. Frankia sp. strain CcI6, isolated from the nodules of Casuarina cunninghamiana trees growing in Egyptian soil, is highly $\mathrm{NaCl}$ tolerant, exhibiting a minimum inhibitory concentration (MIC) value of 1000 mM [14]. Frankia sp. strain CcO1, also from C. cunninghamiana, showed a high tolerance level up to $500 \mathrm{mM} \mathrm{NaCl}$ [3]. Similarly, Frankia sp. strain Ceq1, isolated from C. equisetifolia, is able to withstand up to $500 \mathrm{mM} \mathrm{NaCl}[3,4]$. However, there is great variation in salt tolerance among the different Casuarina associated Frankia isolates, with tolerance values ranging from $100 \mathrm{mM}$ to $1000 \mathrm{mM}[3,14]$. It is of great interest to the scientific community to link the observed tolerance level to the genetic make-up of the strains. To date, more than 38 Frankia genomes have been sequenced and annotated, and 33 of them have been deposited to the NCBI database. The sequenced Frankia strains include Casuarina isolates from a broad range of geographic locations [1522]. The availability of several sequenced Casuarina genomes provides an opportunity to combine phenotypic studies on the strains with comparative genomics and transcriptomics analyses. This study could shed light on known and novel mechanisms of salt tolerance that could help explain the observed disparity in salt tolerance among the different Frankia strains isolated from Casuarina trees.

For many microbes, there are several well-known mechanisms by which they adapt to fluctuations in osmolarity or salt including: (1) re-establishing osmotic balance by accumulating low molecular weight organic compatible solutes [23-25], (2) exclusion of $\mathrm{Na}^{+}$ion from cells via the action of a Na$/ \mathrm{H}^{+}$antiporter and $\mathrm{Na}^{+}$-ATPase [24], (3) altering membrane composition through changes in fatty acid saturation or phospholipid composition to better cope with the changed turgor pressure [26], (4) reactive oxygen species scavenging to prevent the oxidative degradation of lipids, also known as lipid peroxidation [24], (5) restoration of the native folding of proteins through the actions of molecular chaperons [27].

Compatible solutes encompass a restricted range of highly water soluble, osmotically active, low molecular weight amino acids and their derivatives, sugars or sugar alcohols, other alcohols [28] and inorganic cations such as $\mathrm{K}^{+}$[29]. Commonly employed compatible solutes include the sugar trehalose, the amino acids proline, serine and glutamate [30], quaternary ammonium compounds such as glycine betaine and proline betaine [31], polyamines, and organic solutes [30]. The strikingly limited number of compatible solutes used in all forms of life from bacteria to higher organisms reflect the challenge of finding solutes that are compatible with cellular functions. The osmotic function of a compatible solute depends on the degree of methylation and length of the hydrocarbon chain [32]. Evolutionary selection for a compatible solute depends on the osmotic function as well as on other secondary functions such as its contribution towards heat and cold tolerance [33]. Accumulation of compatible solutes helps to avoid external osmolality-triggered water fluxes along the osmotic gradient causing either swelling in hypotonic environments or plasmolysis under hypertonic ones. Osmotic adaptation using compatible solutes is characterized by a minimal requirement for genetic change and a high degree of flexibility in allowing organisms to adapt to wide ranges of external osmolarity [34].

Salt stress can upset the balance between different cellular processes. The uncoupling of different pathways leads to the transfer of high energy electrons to molecular oxygen $\left(\mathrm{O}_{2}\right)$, causing formation of reactive oxygen species, ROS [35]. ROS cause oxidative damage to proteins, DNA and lipids [36]. Oxidative stress results in the oxidative degradation of lipid membranes, also referred to as lipid peroxidation. The cell needs to be equipped with a mechanism for 
dealing with the degradation products, including over 200 types of aldehydes, many of which are highly reactive and toxic [37].

Cells growing in high salt medium also face a loss of intracellular water, which creates an environment of low water activity and high ionic strength inside the cell. Proteins risk permanent unfolding in the resulting intracellular environment. An effective mechanism to counter this is increasing the activities of molecular chaperones which restore protein function and structure [27].

The deleterious effects of salt stress are first experienced by the cell membrane which separates the interior of the cell from the outside environment [38]. Various kinds of stresses including, but not limited to, heat shock, cold shock, osmotic shock and salinity stress cause disruption of membranes, thereby affecting membrane-linked physiological processes such as transport, enzyme activities and signal transduction. Maintaining correct fluidity of the bilayer over a wide range of salinity determines the extent of cell survival during salt stress [39]. Management of the lipid profile in response to salinity involves induction of fatty acid desaturases, which help to synthesize unsaturated fatty acids from saturated fatty acids [40]. The inherent salt tolerance of the organism dictates such responses and is a key factor accounting for the disparity in salt tolerance between organisms [41].

In this study, we attempted to understand the mechanisms of salt-tolerance in Frankia and have taken a comprehensive molecular and genomic approach to address this question. The salt stress tolerance levels for several Frankia strains isolated from Casuarina trees were assayed and two salt-stress tolerant strains (Frankia sp. strain CcI6 and Allo2) which could withstand up to $1000 \mathrm{mM} \mathrm{NaCl}$ and one relatively salt-sensitive strain (Frankia casuarinae strain CcI3), which could withstand only $475 \mathrm{mM} \mathrm{NaCl}$ were identified. The other Casuarina isolates had intermediate levels of salt-tolerance. Comparative genomics were used to link the observed difference in phenotype to the underlying genetic makeup. Transcriptome and proteome profiling of one of the two highly salt-tolerant strains was carried out under salt and osmotic stress conditions to identify genes involved in salt and osmotic stress responses.

\section{Methods}

\section{Frankia strains and growth conditions}

Frankia sp. strain CcI6 [42], Frankia sp. strain Allo2 [43], Frankia sp. strain Thr [44], Frankia sp. strain BMG5.23 [45], Frankia sp. CeD, Frankia sp. CgI82, Frankia casuarinae strain CcI3 [46, 47], Frankia sp. strain BR, Frankia alni strain ACN14a [46, 48], Frankia sp. strain EAN1pec [49], Frankia sp. strain DC12 [50], and Frankia inefficax strain EuI1c [51, 52] were used in this study. For all strains, except for Frankia inefficax strain EuI1c, Frankia sp. strain EAN1pec, Frankia sp. strain ACN14a, and Frankia sp. strain DC12, stock cultures were grown and maintained in $5 \mathrm{mM}$ propionate basal growth medium supplemented with $\mathrm{NH}_{4} \mathrm{Cl}$ as described previously [53]. For the other strains, proportionate as a carbon source was replaced with $20 \mathrm{mM}$ glucose ( $F$. inefficax strain EuI1c and Frankia sp. strain DC12) and $20 \mathrm{mM}$ succinate (Frankia sp. strain EAN1pec and $F$. alni strain ACN14a). Unless specified otherwise, all Frankia cultures were incubated at $28^{\circ} \mathrm{C}$.

\section{Salt sensitivity assay}

The salt tolerance levels of Frankia strains were determined by measuring the total cellular protein content and/or total cellular dry weight after growth under salt or osmotic stress. For total cellular protein determination, a 24-well plate growth assay was used as described previously [14]. Briefly, cells were grown in propionate basal medium with or without $5 \mathrm{mM} \mathrm{NH} \mathrm{NH}_{4} \mathrm{Cl}$ containing different concentrations of $\mathrm{NaCl}[0-1100 \mathrm{mM}]$ or sucrose $[0-1100 \mathrm{mM}]$. For strains ACN14a, Eul1c, DC12 and EAN1pec, propionate was replaced with the appropriate carbon source described above. The inoculum was adjusted to $40 \mu \mathrm{g} / \mathrm{ml}$ of total protein and the plates were incubated at $28{ }^{\circ} \mathrm{C}$ for 14 days. Growth was measured by total cellular protein content as described below. Growth yield was determined by subtracting the protein content of the inoculum.

For total cellular dry weight determination, Frankia strains were inoculated into $25 \mathrm{ml}$ of basal growth medium containing different concentrations of $\mathrm{NaCl}$ or sucrose $[0-1000 \mathrm{mM}]$. The inoculum concentration was adjusted to $40 \mu \mathrm{g} / \mathrm{ml}$ protein. The Frankia cells were grown for 14 days at $28{ }^{\circ} \mathrm{C}$. Growth was measured by total cellular dry weight as described below. Growth yield was determined by subtracting the dry weight of the inoculum.

To evaluate the levels of tolerance, the following two parameters were used: maximum tolerable concentration (MTC) and minimum inhibitory concentration (MIC). The MTC value is the highest concentration of salt which does not affect the growth, while the MIC value is the lowest concentration of salt that inhibits growth.

\section{Protein content and dry weight determination}

Total cellular protein content was measured by the bicinchonic acid (BCA) method [54] per the manufacturer's specifications (Pierce, Rockford, IL, USA) and bovine serum albumin was used as a standard. Cells solubilized in $1 \mathrm{~N} \mathrm{NaOH}$ were boiled at $95{ }^{\circ} \mathrm{C}$ for $10 \mathrm{~min}$ and centrifuged at $13,000 \mathrm{~g}$ for $5 \mathrm{~min}$. Triplicate measurements were made for each sample. Total cellular dry weight was determined using tarred polycarbonate membranes [55]. 


\section{Vesicle induction and nitrogenase activity}

To determine vesicle production and nitrogenase activity, Frankia cells were harvested after 14 days of growth in medium supplemented with $5.0 \mathrm{mM} \mathrm{NH}_{4} \mathrm{Cl}$ and washed three times with buffer containing $20 \mathrm{mM}$ morpholinepropanesulphonic acid (MOPS) and $10 \mathrm{mM} \mathrm{KH}_{2} \mathrm{PO}_{4}$ buffer at $\mathrm{pH}$ 6.8. The washed cells were inoculated into growth medium lacking an external nitrogen source and containing different concentrations of $\mathrm{NaCl}$ or sucrose. The cultures were incubated at $30{ }^{\circ} \mathrm{C}$ for 4 days. The vesicle numbers were determined as described previously [56]. The activity of the nitrogenase enzyme was determined by the acetylene reduction assay as described previously [56].

\section{Accession numbers}

For bioinformatics analysis, genome sequences and their annotations including amino acid and nucleotide FASTA files were obtained from the NCBI database (http:// www.ncbi.nlm.nih.gov) under GenBank accession numbers [NZ_AYTZ00000000.1, NZ_LRTJ00000000.1, NZ_JE NI00000000.1, NZ_JPGU00000000.1, NZ_JDWE00000 000.1, NZ_JPHT00000000.1, NC_007777.1, NC_008278.1, NC_008578.1]. RNA-seq information is available in the NCBI Gene Expression Omnibus database under accession number GSE95217.

\section{Pan genome analysis}

The web platform OrthoVenn [57] was used to identify orthologous gene clusters. OrthoVenn uses a modified version of the heuristic approach named OrthoMCL [58] to identify ortholog groups. An E-value cut off of $1 \mathrm{e}^{-5}$ was used for all-to-all protein similarity comparisons. An inflation value of 1.5 was used for the generation of orthologous clusters using the Markov Cluster Algorithm [59]. To determine single copy orthologs among the most tolerant, moderately tolerant and most sensitive strains, a modified Lerat method was used [60]. As a stringent criterion for homology, only gene pairs representing a bit score value equal to or higher than $30 \%$ of the maximal possible bit score value were considered homologous genes. A Venn diagram in $\mathrm{R}$ was used to construct the four-way Venn diagram of shared CDSs between salt-tolerant (2 strains), moderately salt-tolerant (1 strain), and the relatively salt-sensitive strain.

\section{Average nucleotide identity, average amino acid identity and average genomic distance}

The average nucleotide identity (ANI) and average amino acid identity (AAI) between strains was estimated by using reciprocal best hits (two-way ANI or two-way AAI), as previously described [61]. Genome to genome distance was calculated using the web platform GGDC 2.1 according to the standard operating procedure previously described [62]. GGDC 2.1 BLAST+ was chosen as the alignment method for finding intergenomic matches.

\section{Phylogenetic analysis}

A concatenated maximum parsimony phylogenetic tree was generated from 394 conserved single copy panorthologous genes determined by a modified Lerat method [60]. The rationale for including only singlecopy genes representing species divergences was to minimize potential errors caused by gene duplication. The tree was calculated by determining the ratio of the bit score to the maximal bit score (i.e. protein match against itself). As a stringent criterion for homology, two genes are considered homologous only if the bit score value for the pair is at least $30 \%$ of the maximal bit score. The $30 \%$ cutoff maximized the number of families containing genes, and is optimal for the interspecific identification of homologous sequences [63].

\section{RNA-seq sample preparation and data analysis}

To analyze gene expression of the salt-tolerant strain under salt stressed conditions, RNA-seq analysis was performed on one of the two salt-tolerant strains (CcI6). Cultures were grown for 7 days at $28{ }^{\circ} \mathrm{C}$ in $5 \mathrm{mM}$ propionate, $5 \mathrm{mM} \mathrm{NH}_{4} \mathrm{Cl}$ basal growth medium [51] alone or supplemented with $200 \mathrm{mM} \mathrm{NaCl}$ or sucrose. The bacteria were harvested and the pellets were frozen at $-80^{\circ}$ $\mathrm{C}$ until needed. Total RNA was extracted using a modified RNeasy Midi kit (Qiagen Sciences, Valencia, CA). Frozen bacterial pellets were resuspended in $0.5 \mathrm{~mL}$ TE buffer, $\mathrm{pH} 8$, supplemented with $5 \mathrm{mg} / \mathrm{ml}$ lysozyme and incubated at room temperature for $10 \mathrm{~min}$. RLT buffer (2 ml) supplemented with $1 \mu \mathrm{l} / \mathrm{ml} \beta$-Mercaptoethanol $(\beta-\mathrm{ME})$ was added to each sample and the pellets were homogenized. Subsequently, the RNeasy midi kit procedure was followed as per the manufacturer's recommendation with one major modification: after addition of ethanol to the lysate, the RNeasy mini kit procedure, instead of the RNeasy midi kit procedure, was used. RNA samples were treated with DNase I (New England Biolabs, Ipswich, Massachusetts) per the manufacturer's instructions. RNA was quantified using Qubit RNA assay (Invitrogen) and Nanodrop 2000c spectrophotometer (Thermo Scientific, Wilmington, Delaware) according to manufacturers' specifications. The quality of each RNA sample was determined using the Agilent 2100 Bioanalyzer (Agilent, Santa Clara, CA) according to the Prokaryote Total RNA Nano protocol. RNA quality was represented by RNA integrity number (RIN value), which ranged from 1 to 10 , with 10 representing the most intact RNA. Samples with RIN value greater than or equal to 8 were used for downstream analysis. Ribosomal RNA was removed from 2 to $4 \mu \mathrm{g}$ of total 
RNA by the use of the MicrobeExpress kit (Ambion, Foster City, CA) according to the manufacturer's specifications. The MEGAclear kit (Life Technologies, Carlsbad, CA) was used to remove tRNA according to manufacturer's specifications. cDNA libraries were prepared using the TruSeq RNA Sample Prep Kit (Illumina, San Diego, CA) as described by the manufacturer. The cDNA library was verified for appropriate fragment size (approximately $250 \mathrm{bp}$ ) on an Agilent 2100 Bioanalyzer (Agilent, Santa Clara, CA) according to the DNA 1000 protocol described by the manufacturer. The Qubit dsDNA BR Assay (Invitrogen, Carlsbad, CA) was used to determine the CDNA concentration of each library according to manufacturer's recommendations. Libraries were normalized to $10 \mathrm{nM}$ with $10 \mathrm{mM}$ Tris- $\mathrm{HCl}$, $\mathrm{pH} 8.5$, supplemented with $0.1 \%$ Tween 20 . Illumina sequencing was carried out at Hubbard Genome Center at the University of New Hampshire on an Illumina HiSeq 2500 platform. Reads were separated on adapter assignment and pre-processed through CASAVA 1.8.3. The resulting FASTQ files of sequence reads were processed using CLC Genomics Workbench 9.0 (CLC bio, Cambridge, MD). Adapters were trimmed from reads by searching on the forward and reverse strands. The ends of reads were quality trimmed based on quality scores from a base-caller algorithm using a limit value of 0.05 . The high quality trimmed reads were mapped to Frankia sp. strain CcI6 gene regions. Reads mapping to rRNA operons were excluded from downstream analysis. Mapping parameters were as follows: The maximum number of mismatches allowed was 2 . The minimum length fraction was set so that at least $50 \%$ of the read length aligns to the reference sequence. The minimum fraction of identity between the read and the reference sequence was set at $80 \%$. A read that matched to more than 10 distinct places in the reference was not mapped. If the read matched to multiple distinct places, but below 10 different locations, it was randomly assigned to one of the distinct places. After mapping, the expression level for each gene was tabulated in terms of the unique number of reads mapping to that gene. All RNA-seq experiments were normalized by the total number of reads. A gene was expressed if it had at least one unique sequence read aligned with it. To determine differential gene expression, statistical analysis on proportions was carried out. Two-sided $p$-values for multiple biological replicates were computed using Baggerley's test [64].

\section{Proteome analysis of salt-stressed Frankia sp. strain Ccl6 and Frankia sp. strain $\mathrm{Ccl} 3$}

Cultures were grown for 7 days at $28{ }^{\circ} \mathrm{C}$ in $5 \mathrm{mM}$ propionate, $5 \mathrm{mM} \mathrm{NH}_{4} \mathrm{Cl}$ basal growth medium [53] alone or supplemented with $200 \mathrm{mM} \mathrm{NaCl}$ or sucrose. Frankia mycelium from $50 \mathrm{~mL}$ culture was harvested by centrifugation at
$3,400 \times \mathrm{g}$ for $20 \mathrm{~min}$ and resuspended in $2 \mathrm{~mL}$ of lysis buffer [10 mM Tris- $\mathrm{HCl}$ (pH 7.4), $1 \mathrm{mg} / \mathrm{mL} \mathrm{MgCl}_{2}, 50 \mu \mathrm{g} / \mathrm{mL}$ DNase, $50 \mu \mathrm{g} / \mathrm{mL}$ RNAse, and $50 \mu \mathrm{g} / \mathrm{mL}$ lysozyme]. A French pressure cell was used to lyse the cells at $137,895 \mathrm{kPa}$. Lysed samples were centrifuged at 16,200×g and the supernatant fluid containing soluble proteins was collected. Protein samples were quantified using the Bradford assay [65]. One milligram of soluble protein was precipitated with $10 \%(v / v)$ trichloroacetic acid (TCA) in acetone solution containing $20 \mathrm{mM}$ dithiothreitol (DTT) overnight at $-20{ }^{\circ} \mathrm{C}$. The samples were centrifuged at $16,200 \times \mathrm{g}$ for $30 \mathrm{~min}$ at $4{ }^{\circ} \mathrm{C}$ to pellet the proteins. Traces of TCA were removed from the pellet by washing with $20 \mathrm{mM}$ DTT in acetone. The protein pellet was dissolved in $300 \mu \mathrm{L}$ of rehydration buffer [7 M urea, $2 \mathrm{M}$ thiourea, 5\% (m/v) DTT, 2\% (m/v) Triton X-100, 2\% ( $v / \mathrm{v})$ immobiline $\mathrm{pH}$ gradient (IPG) buffer, $0.02 \%$ bromophenol blue]. The solubilized proteins $(1 \mathrm{mg})$ were used to rehydrate an $11 \mathrm{~cm}$ Immobiline ${ }^{\mathrm{mm}}$ DryStrip $\mathrm{pH}$ gradient strip at $\mathrm{pH} 4-7$ (GE Healthcare Biosciences, Pittsburgh, PA). The protein sample suspended in rehydration buffer was evenly distributed along the lanes of a strip holder and the Immobiline strips were rehydrated with the gel side facing down. DryStrip cover fluid (GE Healthcare Biosciences) was used to cover the strips to prevent evaporation and crystallization of the urea. Rehydration proceeded for $12 \mathrm{~h}$. The rehydrated strips were moved to an Isoelectric focusing (IEF) tray and were positioned with the gel side facing down. Wet ProteomIQ ${ }^{\mathrm{m}}$ IPG Wicks (Proteome Systems, Woburn, Massachusetts) were placed at the anode and the cathode to collect excess salt and other contaminants. The strips and the wicks were covered with $50 \mathrm{~mL}$ of DryStrip cover fluid. The IEF tray was placed in an IsoelectrIQ 2 unit (Proteome Systems, Woburn, MA) and isoelectric focusing was performed under the following settings: $100-10,000 \mathrm{~V}$ gradient for $8 \mathrm{~h}$ and $10,000 \mathrm{~V}$ constant for $8 \mathrm{~h}$. Strips were stored gel side up at $-80^{\circ} \mathrm{C}$ until the second-dimension protein separation step. Strips were washed in $1 \times$ sodium dodecyl sulfate (SDS) running buffer [0.2 M glycine, $25 \mathrm{mM}$ Tris-base, $0.1 \%(\mathrm{~m} / \mathrm{v}) \mathrm{SDS}$ ] and reduction of the proteins was undertaken at room temperature by incubating the strips in SDS equilibration buffer $[50 \mathrm{mM}$ Tris- $\mathrm{HCl}(\mathrm{pH}$ 8.8), $6 \mathrm{M}$ urea, 30\% (v/v) glycerol, $2 \%(\mathrm{~m} / \mathrm{v})$ SDS, trace of bromophenol blue] supplemented with $65 \mathrm{mM}$ DTT for $20 \mathrm{~min}$. Alkylation reaction was performed by washing the strips in deionized water followed by incubation in the same SDS equilibration buffer, supplemented with $135 \mathrm{mM}$ iodoacetamide (IAA), instead of with DTT, for $20 \mathrm{~min}$. The strips were rinsed in $1 \times$ SDS running buffer and loaded on a $12 \%$ SDS - Polyacrylamide gel $(16 \times 16 \mathrm{~cm})$ with the plastic backing against one of the glass plates. Strips were sealed in place with $1 \%(\mathrm{~m} / \mathrm{v})$ agarose in TAE buffer $(40 \mathrm{mM}$ Tris, $20 \mathrm{mM}$ Acetate and $1 \mathrm{mM}$ EDTA, pH 8) supplemented with trace amounts of bromophenol blue for tracking purposes. Proteins were separated by electrophoresis at $100 \mathrm{~V}$ for $30 \mathrm{~min}$ followed by $200 \mathrm{~V}$ for $5 \mathrm{~h}$. Protein spots were 
visualized by incubating the gels in Coomassie Blue stain [0.1\% Coomassie Brilliant Blue R-250, 10\% ( $v / \mathrm{v})$ glacial acetic acid, $50 \%(\mathrm{v} / \mathrm{v})$ methanol] followed by destaining with a destain solution $[10 \%(v / v)$ ethanol, $5 \%(v / v)$ glacial acetic acid]. Differentially expressed spots were excised from the gel and placed in a $0.5 \mathrm{~mL}$ Eppendorf tube. Gel pieces were washed three times by adding fresh $50 \mu \mathrm{L}$ of $25 \mathrm{mM} \mathrm{NH}_{4} \mathrm{HCO}_{3} / 50 \%$ acetonitrile (ACN) each time and vortexing for $15 \mathrm{~min}$. The gel pieces were incubated in $25 \mu \mathrm{L}$ DTT solution $(10 \mathrm{mM}$ DTT in $25 \mathrm{mM} \mathrm{NH} \mathrm{HCO}_{3}$ ) at $56{ }^{\circ} \mathrm{C}$ for $1 \mathrm{~h}$. The supernatant was discarded and $100 \mu \mathrm{L}$ of IAA solution $(55 \mathrm{mM}$ IAA in $25 \mathrm{mM} \mathrm{NH}_{4} \mathrm{HCO}_{3}$ ) was added to the samples. The samples were incubated at room temperature in the dark for $45 \mathrm{~min}$. The supernatant was discarded and samples were washed by adding $100 \mu \mathrm{l}$ of $25 \mathrm{mM} \mathrm{NH}_{4} \mathrm{HCO}_{3}$ and vortexing for $10 \mathrm{~min}$. The supernatant was discarded and gel pieces

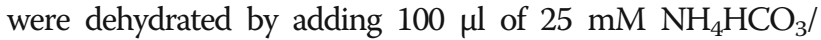
$50 \% \mathrm{ACN}$ solution and vortexing for $10 \mathrm{~min}$. The dehydration step was repeated twice. Gel pieces were completely dried under a speed vacuum for $20 \mathrm{~min}$. Five microliters of trypsin solution ( $40 \mathrm{ng} / \mu \mathrm{L}$ in $25 \mathrm{mM} \mathrm{NH}_{4} \mathrm{HCO}_{3}$ ) were added to the gel pieces. An additional $30 \mu \mathrm{l}$ of $25 \mathrm{mM} \mathrm{NH}_{4} \mathrm{HCO}_{3}$ were added to cover the gel pieces. The trypsin digestion took place at $37^{\circ} \mathrm{C}$ for $4 \mathrm{~h}$. The supernatant from the digestion was transferred to a new tube with $5 \mu \mathrm{l}$ of extraction buffer [50\% $(v / \mathrm{v}) \mathrm{ACN} / 3 \%(v / \mathrm{v})$ acetic acid]. Gel pieces were extracted twice by adding $35 \mu \mathrm{l}$ of extraction solution and vortexing for $20 \mathrm{~min}$. Extracts were combined and dried in a speed vacuum. Extracted peptides were resuspended in $7 \mu \mathrm{l}$ of $0.1 \%$ formic acid. Samples were analyzed using liquid chromatography - mass spectrometry (LC-MS) and LC-MS/MS analysis. An aliquot of the digestion mixture $(1 \mu \mathrm{L})$ was used for LC separation using the Ultimate 3000 RSLCnano UHPLC system with an autosampler (Dionex Corporation, Sunnyvale, California). The eluent was ionized by a nanoelectrospray ionization source of an LTQ Orbitrap XL mass spectrometer (Thermo Scientific, Waltham, Massachusetts). LCMS data were acquired in an information-dependent acquisition mode, cycling between a MS scan (m/z 310-2000) acquired in the Orbitrap, followed by low-energy collisioninduced dissociation (CID) analysis in the linear ion trap. The centroid peak lists of the CID spectra were generated by PAVA [66] and searched against a database that consisted of the National Center for Biotechnology Information (NCBI) protein database, to which a randomized version had been concatenated, using Batch-Tag, a program in the University of California-San Francisco Protein Prospector version 5.10.15. A precursor mass tolerance of $15 \mathrm{ppm}$ and a fragment mass tolerance of $0.5 \mathrm{Da}$ were used for the protein database search. Protein hits were reported with the following parameters: a Protein Prospector protein score of $\geq 22$, peptide score $\geq 15$, and $E$ value for protein $\leq 0.01$ [67]. This set of protein identification parameters threshold did not return any substantial false-positive protein hits from the randomized half of the concatenated database. Test samples were compared with corresponding control samples using the Search Compare program.

\section{Quantitative reverse transcription PCR (qRT-PCR)}

The same RNA samples used for RNA sequencing were also used for qRT-PCR. The RNA (400 ng) was transcribed into cDNA using the GoScript ${ }^{\mathrm{tm}}$ Reverse Transcriptase (Promega, Madison, Wisconsin) according to the manufacturer's instructions. The cDNA was quantified by a Nanodrop 2000c spectrophotometer, diluted to $10 \mathrm{ng} / \mu \mathrm{L}$ working stocks in RNase-free $\mathrm{H}_{2} \mathrm{O}$, and stored at $-20{ }^{\circ} \mathrm{C}$ until used. Amplification and detection of gene expression were performed using Agilent MP3000 qPCR system (Agilent Technologies, Santa Clara, California). The primers used for these experiments are listed in Additional file 1. Each primer sequence was blasted against the Frankia sp. strain CcI6 genome to ensure specificity to the target gene. Standard curves were generated using Frankia sp. strain CcI6 genomic DNA and each primer set to test primer efficiency before use. The qRT-PCR experiments were designed in such a way that gDNA contamination could be kept under check. The rpsO (CCI6_RS04555) gene was used as the normalizer for all qRT-PCR experiments. The qRT-PCRs were done using 50 ng template cDNA, primer mix $(0.3 \mu \mathrm{M})$, and SYBR Green PCR Master Mix (Applied Biosystems, Carlsbad, California) in a $25 \mu \mathrm{L}$ total reaction volume. The following thermal cycler parameters were used: (i) $95{ }^{\circ} \mathrm{C}$ for $15 \mathrm{~min}$; (ii) 40 cycles of $95^{\circ} \mathrm{C}$ for $15 \mathrm{~s}$ and $60^{\circ}$ $\mathrm{C}$ for $30 \mathrm{~s}$; and (iii) 1 thermal disassociation cycle of $95^{\circ}$ $\mathrm{C}$ for $60 \mathrm{~s}, 55^{\circ} \mathrm{C}$ for $30 \mathrm{~s}$, and incremental increases in temperature to $95^{\circ} \mathrm{C}$ for $30 \mathrm{~s}$. Reactions were performed in triplicate. The $\Delta \Delta \mathrm{Ct}$ method [68] was used to calculate relative expression (fold changes).

\section{Results}

Strains Cc16 and Allo2 are highly salt tolerant

The salt tolerance levels for Frankia strains isolated from Casuarina hosts were measured and compared to the levels found for Frankia strains isolated from nonCasuarina hosts. Strains CcI6 and Allo2 were highly salt-tolerant and exhibited a $\mathrm{NaCl} \mathrm{MIC}$ value up to $1000 \mathrm{mM}$ (Fig. 1a). Strain CcI3 was the least salttolerant having a MIC value around $475 \mathrm{mM}$. The other Casuarina isolates had intermediate levels of tolerance with MIC values ranging from $650 \mathrm{mM}$ to $750 \mathrm{mM}$. In general, Casuarina isolates had a higher level of salt tolerance compared to other Frankia isolates, but this higher level of tolerance was not extended to osmotic stress (Fig. 1a). Here, the other Frankia isolates exhibited higher levels of tolerance to osmotic stress compared to the Casuarina isolates. 


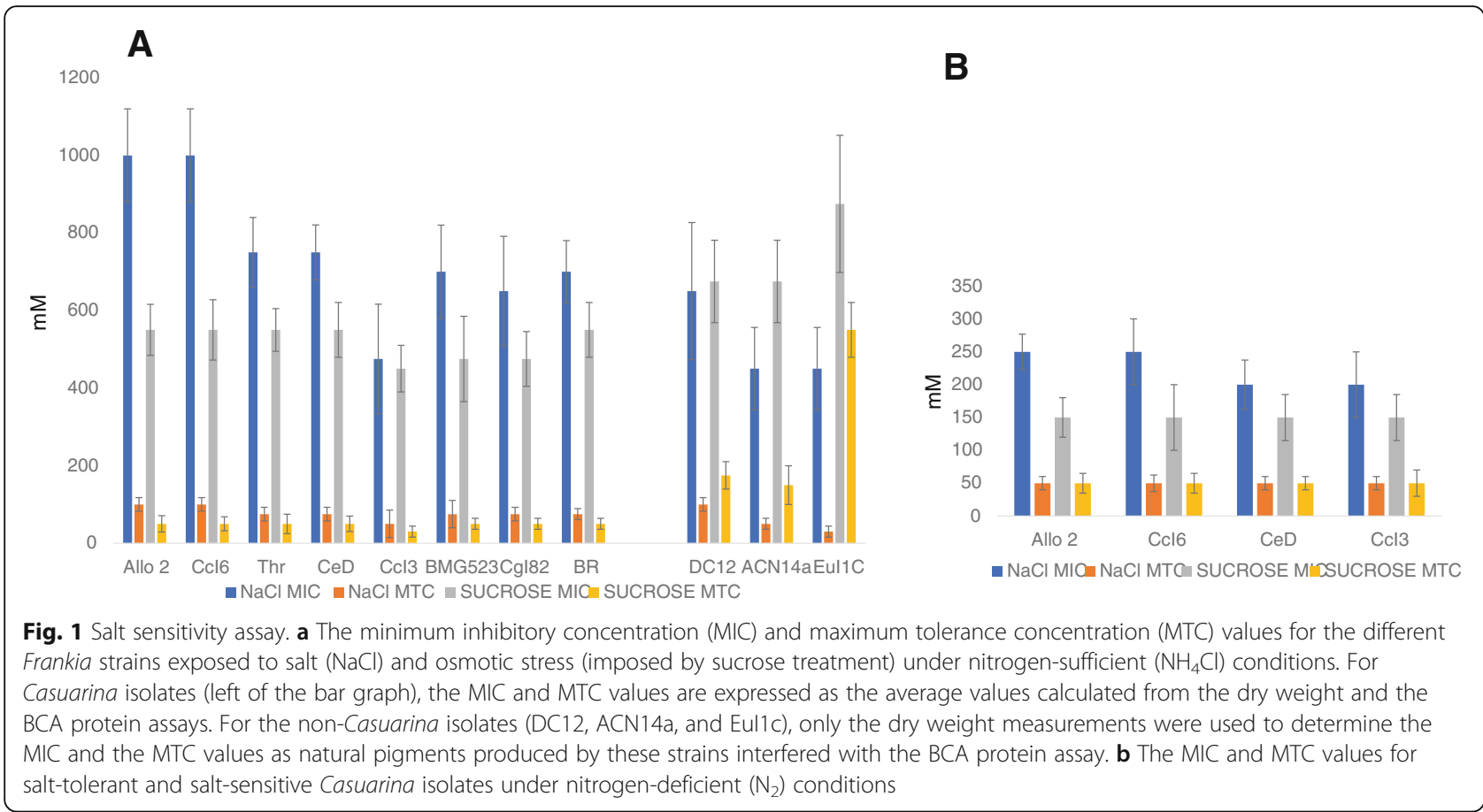

Salt tolerance is highly dependent on the external supply of nitrogen

Under nitrogen deficient conditions, the level of salt tolerance by Frankia strains (including the most salt-tolerant isolates) dramatically decreased (Fig. 1b). For strains CcI6 and Allo2, the $\mathrm{NaCl} \mathrm{MIC} \mathrm{value} \mathrm{decreased} \mathrm{from} 1000 \mathrm{mM}$ under nitrogen sufficient conditions to $250 \mathrm{mM}$ under nitrogen deficient conditions. The observed differences in salt tolerance levels between the salt-tolerant and saltsensitive strains also substantially decreased under nitrogen deficient conditions (Fig 1b). Vesicle formation and nitrogenase activities were also affected by salt stress. Nitrogenase activity was more severely affected (Additional file 2). When the strains were grown in a medium containing greater than $200 \mathrm{mM} \mathrm{NaCl}$, nitrogenase activity was drastically reduced (Additional file 2).

\section{Genomic characteristics of Frankia strains isolated from Casuarina trees}

Table 1 presents the genomic features of Frankia strains isolated from Casuarina trees. The size and $\mathrm{G}+\mathrm{C}$ content of the genomes used in this study ranged from approximately 5 to $5.6 \mathrm{MB}$ and 69.3 to $70.1 \% \mathrm{G}+\mathrm{C}$, respectively. Strain $\mathrm{CcI} 3$ had the highest number of CDSs (4327), while strain CeD had the lowest number of CDSs (3807).

We obtained a phylogeny of the Frankia strains from a set of 394 conserved non-duplicated orthologs (Fig. 2a). As expected, the eight Casuarina strains grouped together and were distinct from the closely related Cluster-1a strain ACN14a, which was isolated from Alnus trees. Strains CcI6 and Allo2 showed close similarity and grouped together, while strains CeD and BMG5.23 showed the least similarity with other strains.

Average nucleotide identity, average amino acid identity, and genome to genome distance

The average nucleotide identity between any pair of Frankia strains isolated from Casuarina trees was greater than 99\%. In contrast, the average nucleotide identity between any Casuarina isolate and the closely-related strain ACN14a was less than 85\% (Fig. 2b). Similar results were obtained for the average amino acid identity and genometo-genome distance values. Based on DNA-DNA hybridization (DDH) prediction by GGD 2.1, at $p=0.05$ level, any two Casuarina isolates have at least 70\% DDH value, the cutoff point for species delineation. On the other hand, in a pairwise comparison with strain ACN14a, none of the Casuarina isolates had DDH value greater than or equal to $70 \%$ at $p=0.05$ level.

\section{Pan-genome analysis reveals a high abundance of singletons among all of the strains}

Pan-genome analysis was performed by orthologous clustering using OrthoVenn. OrthoVenn utilizes OrthoMCL to perform an all-against-all BLASTP alignment and identify putative orthology and paralog relationships with the Inparanoid algorithm.

The OrthoVenn analysis of the six Casuarina isolates selected after the salt sensitivity assay revealed 3278 pan-orthologous gene clusters, of which 3246 were single copy pan orthologous gene clusters (Fig. 3a). Pairwise 
Table 1 Genomic features of Frankia strains isolated from Casuarina trees

\begin{tabular}{|c|c|c|c|c|c|c|c|}
\hline & \multicolumn{7}{|c|}{ Frankia sp. strains isolated from Casuarina trees } \\
\hline & $\mathrm{Ccl} 6$ & Allo2 & $\mathrm{CeD}$ & Thr & $\mathrm{Br}$ & $\mathrm{Ccl} 3$ & BMG5.23 \\
\hline Chromosome size(Mb) & 5.58 & 5.35 & 5.00 & 5.31 & 5.23 & 5.43 & 5.27 \\
\hline GC \% & 69.3 & 70.0 & 70.0 & 70.0 & 70.0 & 70.1 & 69.9 \\
\hline $\mathrm{N}_{50}(\mathrm{bp})$ & 103,000 & 96,900 & 73,600 & 71,600 & 60,200 & 543,3628 & 64,900 \\
\hline CDS & 4280 & 4224 & 3857 & 4209 & 4220 & 4327 & 4114 \\
\hline rRNA & 9 & 8 & 6 & 5 & 4 & 6 & 9 \\
\hline tRNAs & 45 & 45 & 45 & 45 & 45 & 45 & 48 \\
\hline \#Scaffolds & 136 & 110 & 120 & 169 & 180 & 1 & 166 \\
\hline \#Contigs & 155 & 133 & 154 & 184 & 180 & 1 & 191 \\
\hline Reference & [17] & [21] & [18] & [16] & [19] & [20] & [15] \\
\hline
\end{tabular}

comparison of the genomes showed that strains CcI6 and Allo2 shared the highest number of unique clusters (132), not found in the other strains (Additional file 3). No genome had more than 2 clusters unique to itself, but all had many singletons (Fig. 3b) suggesting that there was insufficient time for gene duplication events to occur after the appearance of singletons. Among the six Casuarina isolates, strain BMG5.23 had the highest number of singletons (160). About $30 \%$ of the singletons in any one strain were hypothetical proteins. Singletons generally occur dispersed within the genome, suggesting they were acquired independently. However, in strain BMG5.23, singletons seem to be clustered in the same region. The singletons in strain BMG5.23 had varying GC contents and the fact that at least some of them cooccur in the same region suggests that there might be hot spots for insertion.
The two salt-tolerant strains contain many hypothetical proteins absent in the other strains

While genomic comparison of the salt-tolerant strains (CcI6 and Allo2) against the entire Casuarina-associated strains gave insight on the strain difference, this analysis included multiple gene copies. Comparison of the single copy orthologous gene clusters between the salt-sensitive isolate (CcI3), the moderately salt-tolerant strain (CeD), and the two highly salt-tolerant strains (CcI6 and Allo2) was also performed using the Lerat program. All four strains shared 2919 single copy core genes (Fig. 4a). The two highly salttolerant strains contained 153 single copy orthologous genes that were not shared with the moderately-tolerant and salt-sensitive strains (Additional file 3). Both highly salt-tolerant strains and the moderately-tolerant strain shared 88 single copy genes that were not present in the salt-sensitive strain. Figure $4 \mathrm{~b}$ shows the distribution of

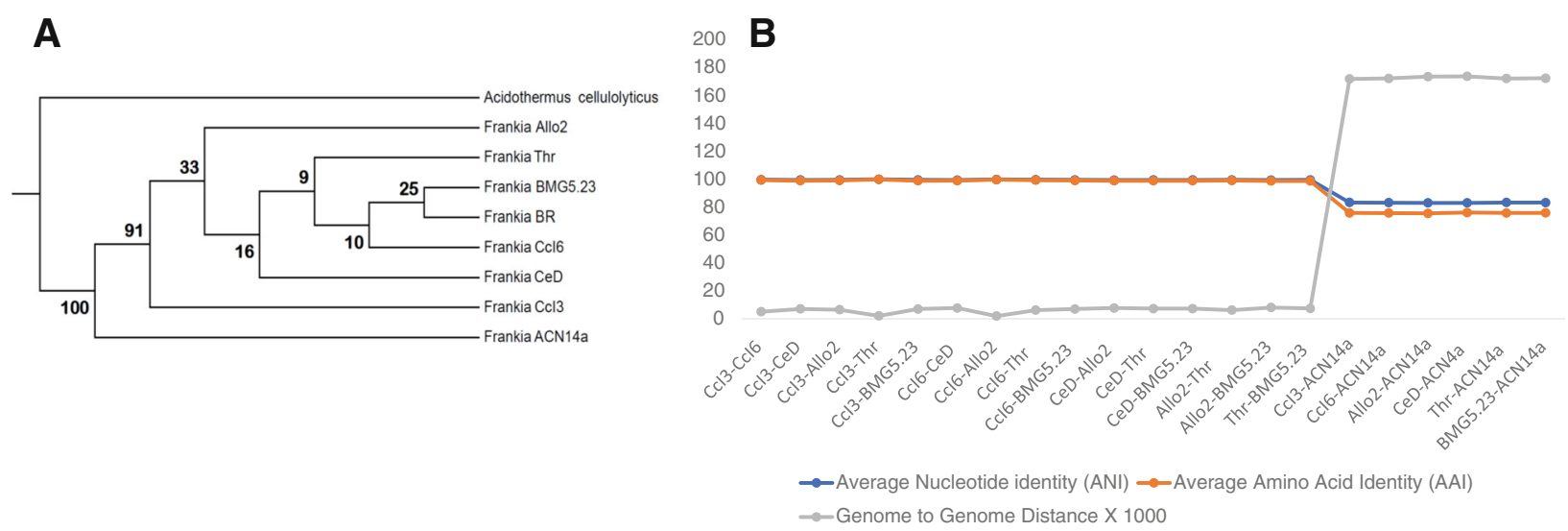

Fig. 2 Genomic taxonomy of Frankia strains isolated from Casuarina spps. a Concatenated phylogenetic affiliation of 394 maximum-parsimony trees for amino acid sequences of orthologs among all of the genomes including Casuarina isolates, F. alni strain ACN14a isolated from Alnus and Acidothermus celloluyticus, which was used as an outgroup. The numbers on the branches represent the percent confidence of speciation of a given branch. b AAl, ANI, and genome to genome distance (multiplied by 1000) values for the different Casuarina isolates and F. alni strain ACN14a isolated from Alnus which is included for comparison. Genome to genome distance was determined by GGDC as a function of sum of all identities found in HSPs divided by overall HSP length. GGDC2 BLAST+ was used as the alignment method for finding intergenomic matches 


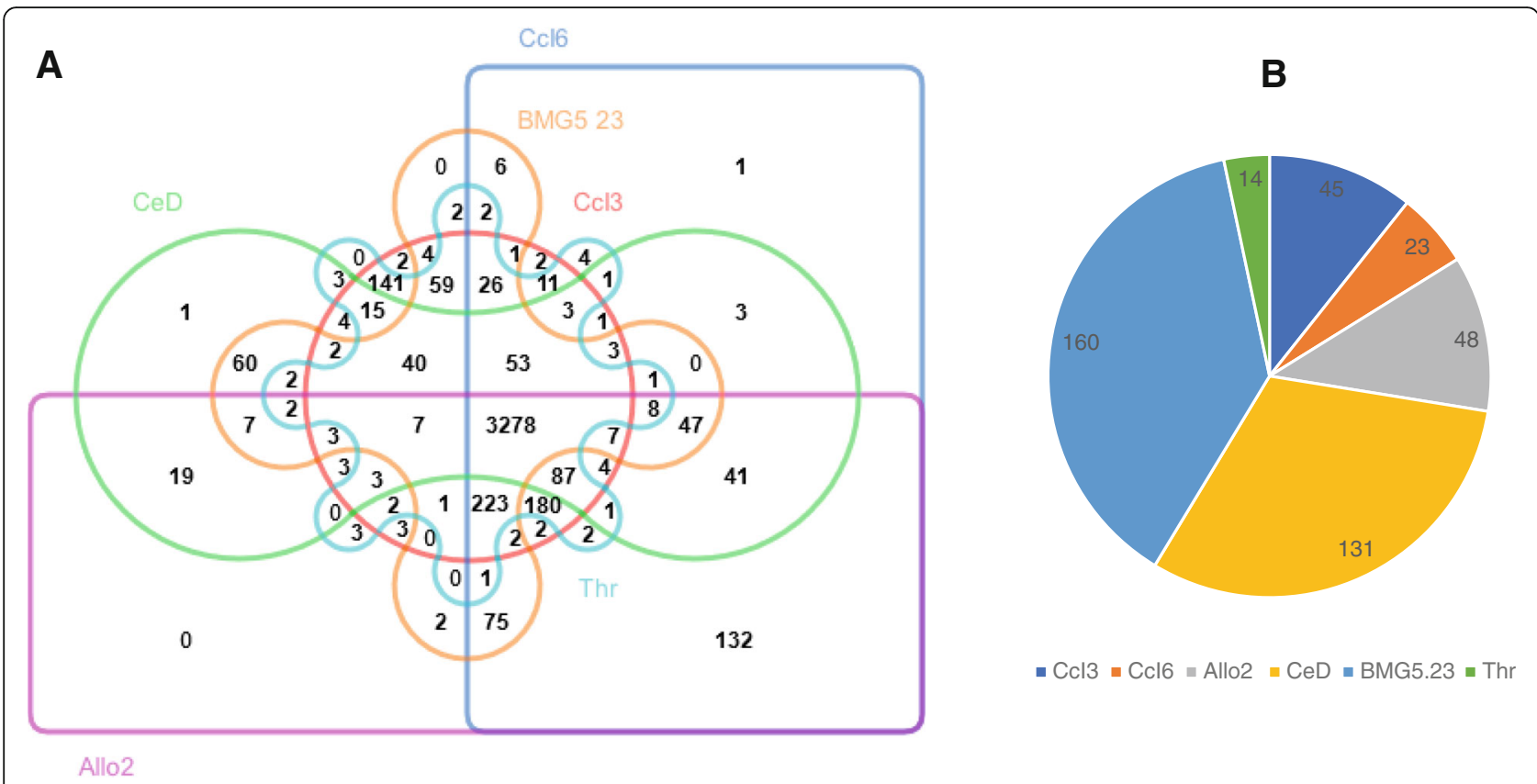

Fig. 3 Pangenome overview of six Frankia strains isolated from Casuarina trees. a Six-way Venn diagram showing shared and specific gene clusters among the Casuarina isolates as determined by OrthoVenn. An E-value cutoff of $1 \mathrm{e}^{-5}$ was used for protein similarity search and inflation value of 1.5 was used for the generation of orthologous clusters using the Markov Cluster Algorithm. b Number of singletons identified in each Casuarina isolate

genes found in the two highly salt-tolerant strains into Cluster of Orthologous Groups of protein (COG) functional categories. Among the 153 unique genes found in the two salt-tolerant strains, 114 of the genes were annotated as hypothetical proteins. However, re-annotation using the RPSBLAST program on the COG database (prokaryotic proteins) revealed only 99 hypothetical proteins. The three COG categories that were highly represented among the unique genes found only in the two highly salttolerant strains were: (COG R) general function prediction

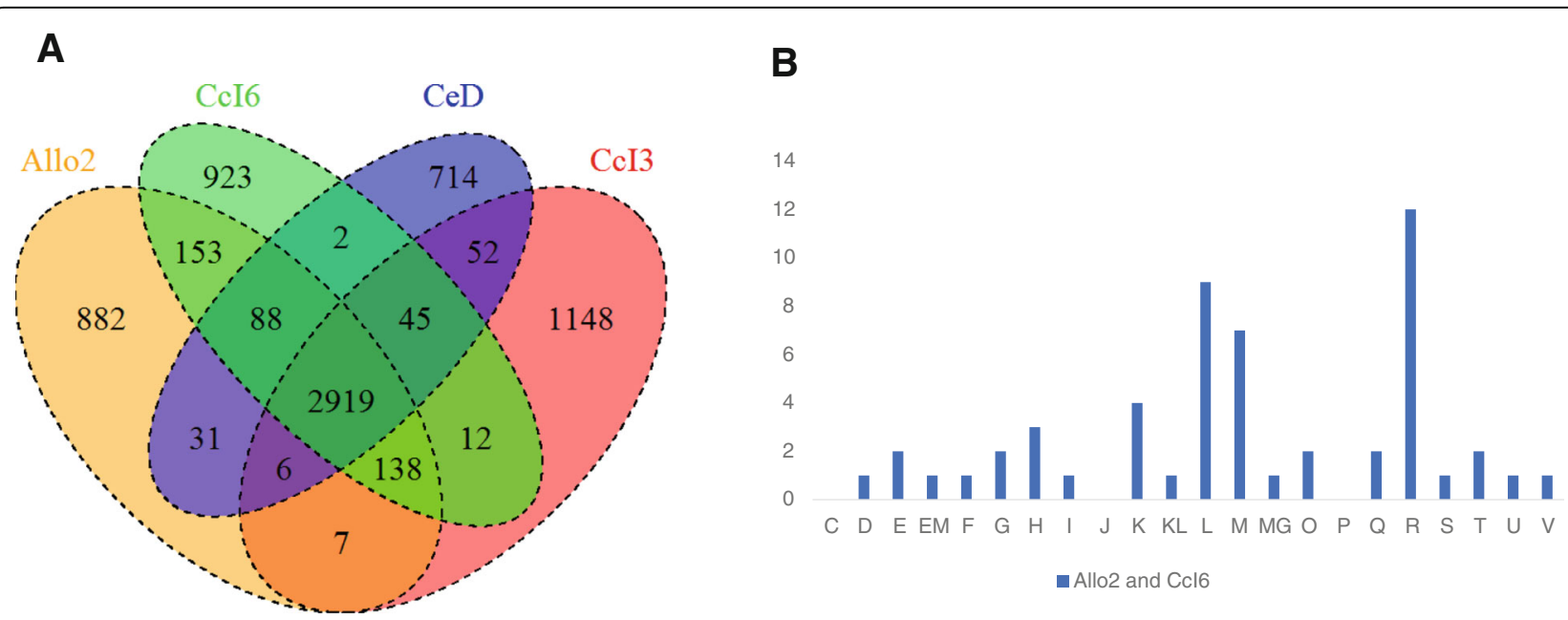

Fig. 4 Pangenome analysis of single-copy genes (a) Shared and specific single-copy orthologous CDSs among the highly salt-tolerant (Allo2 and Cc16), the moderately salt-tolerant (CeD), and the salt sensitive (Ccl3) strains. b Distribution of the 153 single-copy genes specific to the two highly salt-tolerant strains into functional COG categories: C, energy production and conversion; D, cell division and chromosome partitioning; E, amino acid transport and metabolism; F, nucleotide transport and metabolism; G, carbohydrate transport and metabolism; H, coenzyme metabolism; I, lipid transport and metabolism; $\mathrm{J}$, translation, ribosomal structure and biogenesis; $\mathrm{K}$, transcription; L, DNA replication, recombination and repair; $\mathrm{M}$, cell wall/membrane biogenesis; $\mathrm{O}$, posttranslational modification, protein turnover, chaperones; $\mathrm{P}$, inorganic ion transport and metabolism; Q, secondary metabolite biosynthesis, transport and catabolism; R, general function prediction only; S, function unknown; T, signal transduction mechanisms; $U$, intracellular trafficking and secretion; and V, defense mechanisms. Proteins that could be classified to more than one category are represented by two letters 
only; (COG L) DNA replication, recombination, and repair; and (COG $\mathrm{M}$ ) cell wall/membrane envelope biogenesis. Tolerant-strain-specific genes assigned to the cell wall/ membrane biogenesis (COG M) category include genes encoding glycosyl transferases, proteins involved in cellulose synthesis, D-alanine: D-alanine ligase (Ddl), and a predicted nucleoside-diphosphate-sugar epimerase. Glycosyl transferases allow for a more flexible response to environmental stress. In tobacco, ectopic expression of a glycosyl transferase (UGT85A5) leads to enhanced salt tolerance [69]. Ddl is involved in the D-alanine branch of peptidoglycan biosynthesis. Mutation in a D-alanine-D-alanine ligase of Azospirillum brasilense Cd results in an overproduction of exopolysaccharides and decreased tolerance to saline stress [70].

Another COG functional category that was represented among the genes found only in the tolerant strains was coenzyme metabolism. Two genes encoding hypothetical proteins (CCI6_RS06345, CCI6_RS15790) with ubiquinone synthesis-related methyl transferase domains and a hypothetical protein with a geranylgeranyl pyrophosphate synthase domain (CCI6_RS02885) were among the tolerant strain-specific genes assigned to the coenzyme metabolism functional category. Geranylgeranyl pyrophosphate (GGPS) synthase catalyzes formation of geranylgeranyl pyrophosphate (GGP), which is a key step in the biosynthetic pathway of carotenoïds and many other terpenes [71].

\section{Salt-tolerant and -sensitive strains contain the same set of classical salt-tolerance genes}

Many of the known salt tolerance mechanisms are also osmotic stress response mechanisms or vice versa. The Casuarina associated Frankia genomes were data mined for the presence of these known osmotic/salt tolerance mechanisms.

All of these genomes had a $k d p F A B C D E$ operon encoding the membrane-associated P-type ATPase, Kdp-ATPase $(k d p F A B C)$, involved in $\mathrm{K}^{+}$uptake and a two-component regulatory system $(k d p D E)$, which regulates the expression of $k d p F A B C$ [72]. The Kdp system plays a role in ion homeostasis and adaptation to osmotic stress. Both tolerant and sensitive strains also contained the Trk system, which is the predominant uptake system in medium containing more than $1 \mathrm{mM} \mathrm{K}^{+}$. All of the Casuarina associated Frankia genomes lacked mechanisms for de novo synthesis or uptake of glycine betaine. However, all of the Casuarina isolates possessed the capacity for the biosynthesis of the important osmo-protectant proline. Three pathways for the synthesis of trehalose, an effective osmolyte, were also present in all the Casuarina associated Frankia genomes. The first pathway (the TreY-TreZ pathway) synthesizes trehalose from glycogen-like alpha $(1->4)$-linked glucose polymers. The second pathway (the TreS pathway) synthesizes trehalose from maltose, while the third pathway, the OtsA-OtsB pathway, utilizes glucose-6-phosphate and UDP-glucose to synthesize trehalose through a twostep enzymatic process involving trehalose-6-phosphate synthase (OtsA) and trehalose-6-phosphate phosphatase (OtsB). All Casuarina isolates contained the asnO-ngg cluster putatively involved in the synthesis of $\mathrm{N}$ acetylglutaminylglutamine amide (NAGGN), a dipeptide identified as an osmolyte in a few bacteria. The first step of the reaction involves the $\mathrm{N}$-acetylation of a glutamine residue and the subsequent dipeptide bond formation between this residue and a second L-glutamine residue in a reaction catalyzed by Ngg. In the second step of the reaction catalyzed by $\mathrm{AsnO}$, an amide group is transferred from a free L-glutamine molecule to the second L-glutamine residue of NAGG to produce NAGGN. Just like most other genomes containing the asnO-ngg cluster, the genomes of all Casuarina isolates encode a dipeptidase immediately downstream of the $n g g$ gene. A possible role for such a peptidase could be balancing of the NAGGN pool during adaptation to osmotic fluctuations. The identities between AsnO from Casuairna isolates and the one found within other bacterial species was high (greater that 60\%) whereas the putative Ngg protein from Casuarina isolates had low identity (< $15 \%$ ) with Ngg proteins identified in other species. In contrast to the asnO-ngg organization found within other genomes, the $a s n O$ and the $n g g$ genes in the Casuarina isolates were not contiguous, but had the dipeptidase gene between them.

\section{Transcriptome analysis of the highly salt-tolerant strain (Ccl6) reveals hundreds of genes that are responsive to salt stress}

After identifying unique genes in the two highly salt tolerant strains through comparative genomics, we attempted to identify the role of these unique genes in the response to salt and osmatic stress. This was done by transcriptome profiling of strain CcI6, one of the two highly salt-tolerant strains, under salt or osmotic stress and comparing to the profile under control conditions (no stress). Strain CcI6 was exposed to either no stress, salt stress or osmotic stress for 7 days and the transcriptome profile was analyzed using RNA-seq. Two biologically independent experiments were performed for each condition. After sequencing of the libraries, an average of 17 million, 9.5 million, and 19.8 million reads were obtained under the control, salt stress, and osmotic stress conditions, respectively. CLC Genomics Workbench 9.0.1 was used to map the reads to the annotated Frankia sp. CcI6 genome. An average of 2.14 million, 0.85 million, and 2.5 million read pairs could be unambiguously mapped to the CDSs for the reference condition, for the salt stress condition, and for the osmotic stress condition, respectively. Based on the mapped reads, coverage for the samples were $42 \times$, 
$17 \times$, and $100 \times$ for the control, salt stress and osmotic stress conditions, respectively.

Transcriptome analysis revealed that a total of 214 and 226 genes were up-regulated and 303 and 167 genes were down-regulated under salt and osmotic stress, respectively. Thirty-five up-regulated and 65 downregulated genes were found in common with both conditions (Fig 5). The complete list of differentially expressed genes is provided in Additional file 4. Among the genes up-regulated under both salt and osmotic stress were hypothetical proteins, proteins involved in cell wall/ membrane biogenesis functions and the following transport proteins: an $\mathrm{ABC}$-type $\mathrm{Fe} 3+$ hydroxamate transport system, periplasmic component (CCI6_RS09145) and ABC-type $\mathrm{Fe} 3+$ siderophore transport system, permease component (CCI6_RS09155). This result suggests that increased iron uptake is part of the general response to salt and osmotic stresses. Increased iron uptake under salt stress has been reported for Bacillus subtilis [73].

A total of 179 genes were only up-regulated under salt stress, while 191 genes were only up-regulated under osmotic stress (Fig 5). The functional category analysis of the up-regulated and down-regulated genes showed that more than $60 \%$ and $61 \%$ of the genes, respectively, were assigned into a COG functional category (Fig. 6b, d). Unassigned genes were mainly comprised of hypothetical proteins. COG functional categories highly represented among up-regulated genes included: (1) general function prediction only (COG R), energy production and conversion (COG C), amino acid transport and metabolism (COG E) [Fig 6a]. Among the up-regulated genes, $3.7 \%$ and $5.5 \%$ of the genes were general function prediction only (COG R) genes specific to salt and osmotic stress, respectively. General function prediction only (COG R) genes up-regulated under both salt and

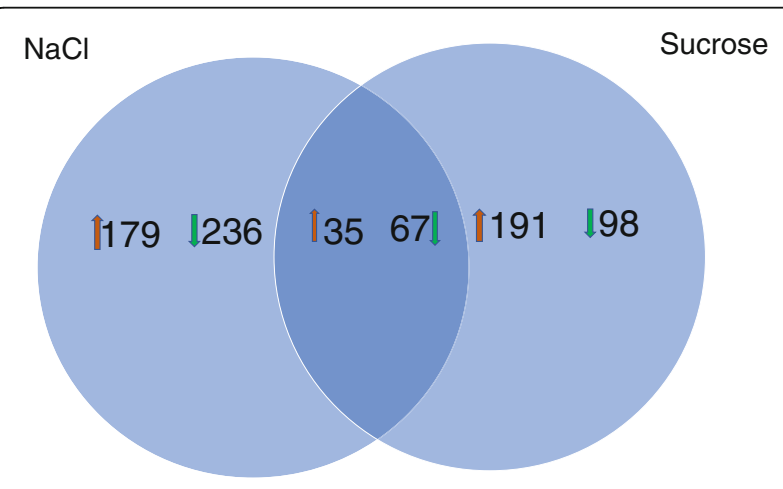

Fig. 5 Global gene expression responses following salt and osmotic stress. Venn diagram showing the extent of overlap between genes differentially expressed under salt and osmotic stress in the salt-tolerant strain Frankia sp. Ccl6. The arrows indicate the number of upregulated and down-regulated genes under salt and osmotic stress. The intersection indicates the number of differentially expressed genes under both conditions osmotic stress constituted $0.5 \%$ of the total up-regulated genes. A total of $2 \%$ and $1.2 \%$ of the up-regulated genes were cell wall/membrane biogenesis (COG M) genes specific to salt and osmotic stress, respectively (Fig 6a). One percent of the up-regulated genes comprised of cell wall/membrane biogenesis (COG M) genes up-regulated under both salt and osmotic stress conditions. Energy production and conversion (COG C) genes comprised $4.7 \%$ of the total up-regulated genes. Among the upregulated genes assigned to COG C, roughly $23 \%$ were specific to salt stress while $65 \%$ were specific to osmotic stress and $12 \%$ were common to both salt and osmotic stress conditions. Functional categories that are highly represented among down-regulated genes include transcription (COG K), general function prediction only (COG R), and function unknown (COG S). Salt stressspecific and osmotic stress-specific transcription (COG K) genes constitute $2 \%$ and $0 \%$, respectively of the down-regulated genes. Transcription (COG K) genes that are down-regulated under both salt and osmotic stress conditions make up $1.5 \%$ of the total downregulated genes.

We validated the RNA-seq data by performing quantitative reverse transcription PCR (qRT-PCR) on 11 genes selected from the RNA-Seq analysis (Table 2). A high degree of correlation $(R=0.95)$ was observed between the normalized values of the fold change from the qPCR data and the normalized fold change values from the RNA-Seq data.

\section{Many hypothetical proteins that are unique to the tolerant strain were up-regulated under salt stress}

Under osmotic stress, an acetyl transferase with general function prediction only and 7 hypothetical proteins that are unique to the two salt-tolerant strains were upregulated. Under salt stress, a zinc peptidase, ADPheptose:LPS heptosyltransferase, and 5 hypothetical proteins unique to the tolerant strains were up-regulated (Table 3). One hypothetical protein (CCI6_RS13590) was up-regulated under both salt and osmotic stress conditions. In a study of differentially expressed genes in salttolerant and salt sensitive varieties of rice, zinc peptidase was among the 50 highest responsive genes in the salttolerant variety [74].

\section{Versatile responses of transcription factors}

Not surprisingly, COG K (transcription) genes were highly represented in the transcriptome of strain CcI6 under salt stress (Additional file 4). Under salt stress, 6 genes (CCI6_RS12535, CCI6_RS20460, CCI6_RS18600,CCI6_R S15305, CCI6_RS01570, CCI6_RS12900, CCI6_RSO2550) encoding transcriptional factors from the GntR, TetR, and LysR, and the Crp/Fnr families and 6 genes (CC I6_RS00475, CCI6_RS02550, CCI6_RS10900, CCI6_RS1 


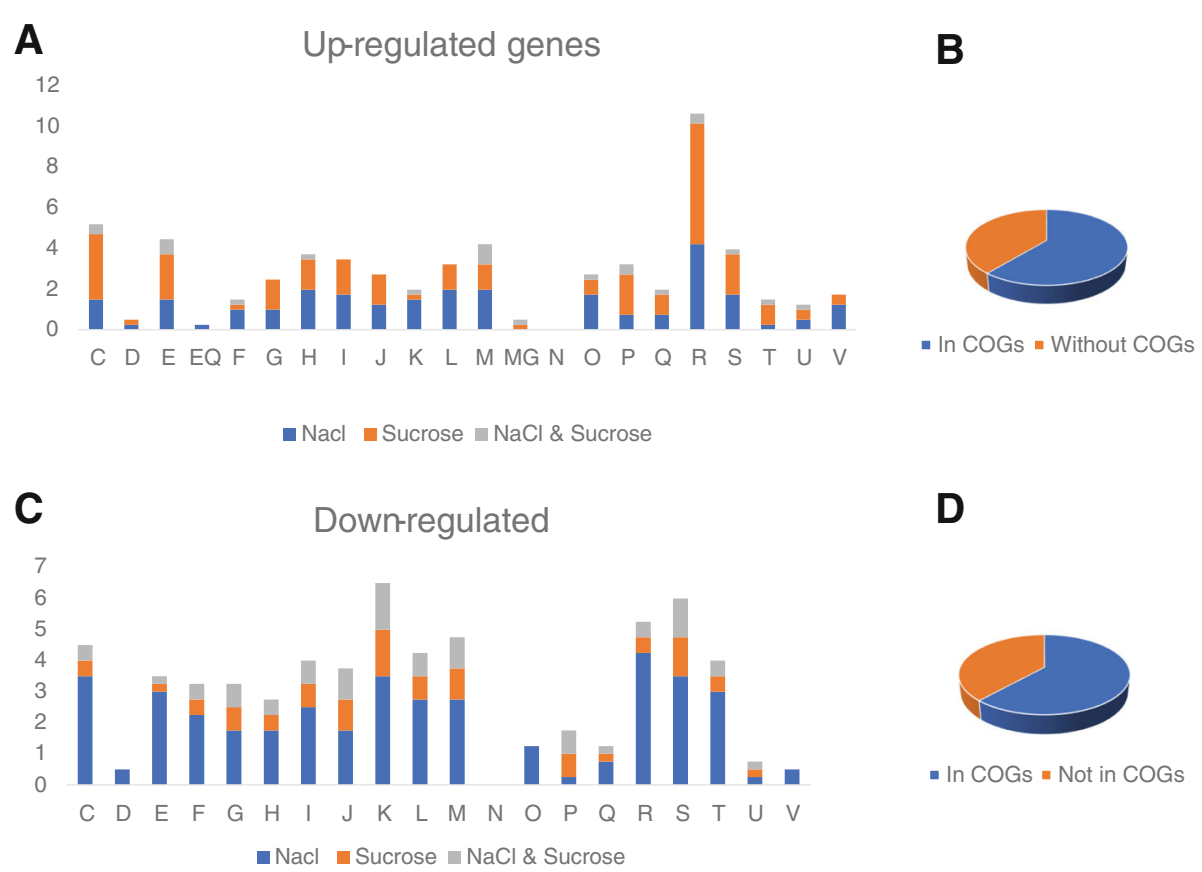

Fig. 6 The percentage of the differentially expressed genes that fell within the various Clusters of Orthologous Gene (COG) categories. The columns are labelled as follows: C, energy production and conversion; D, cell division and chromosome partitioning; E, amino acid transport and metabolism; F, nucleotide transport and metabolism; G, carbohydrate transport and metabolism; H, coenzyme metabolism; I, lipid transport and metabolism; J, translation, ribosomal structure and biogenesis; $\mathrm{K}$, transcription; L, DNA replication, recombination and repair; $\mathrm{M}$, cell wall/membrane biogenesis; $\mathrm{N}$, cell motility; O, posttranslational modification, protein turnover, chaperones; $\mathrm{P}$, inorganic ion transport and metabolism; Q, secondary metabolite biosynthesis, transport and catabolism; R, general function prediction only; S, function unknown; T, signal transduction mechanisms; $\mathrm{U}$, intracellular trafficking and secretion; and $\mathrm{V}$, defense mechanisms. For each condition, the number of up-regulated (or down-regulated) genes in each COG category was expressed as a percentage total number of upregulate or down-regulated genes, respectively. a Up-regulated genes. b Fraction of up-regulated genes that are assigned to COG categories. c Down-regulated genes. $\mathbf{d}$ Fraction of down-regulated genes that are assigned to COG categories

1405, CCI6_RS17055, CCI6_RS21525) encoding transcriptional regulators from Crp/Fnr and LuxR families were up-regulated under salt and osmotic stress, respectively. One transcriptional regulator belonging to the $\mathrm{Crp} / \mathrm{Fnr}$ family (CCI6_RS02550) was up-regulated under both salt and osmotic stress conditions. Transcriptional factors belonging to GntR, TetR, LysR, and the Crp/Fnr families have been implicated previously in several stress responses including heat and osmotic shock [75]. In addition, only one (CCI6_RS19210) of the 12 sigma factors present in strain CcI6 was up-regulated under salt stress, while another one, an extracytoplasmic stress sigma factor (CCI6_RS15595), was down-regulated under salt stress conditions.

\section{Salt stress up-regulated several genes involved in peptidoglycan modification}

Two genes encoding polysaccharide deacetylases (CCI6_RSO3540, CCI6_RS11155) were up-regulated under salt stress, but were unchanged under osmotic stress (Additional file 4). In Bacillus anthracis, a polysaccharide deacetylase plays a role in the adaptation of the bacteria to a high salt environment [76]. Under salt stress only, 4 glycosyl transferases (CCI6_RS10910, CCI6_RSO7965, CCI6_RS21895, CCI6_RS01640) associated with cell wall/membrane biogenesis showed more than 4-fold increase. Similarly, under osmotic stress, 4 glycosyl transferases associated with cell wall/membrane biogenesis (CCI6_RSO2325, CCI6_RS10910, CCI6_R S11195, CCI6_RS11220) were up-regulated. One of the above glycosyl transferase genes (CCI6_RS10910) showed a statistically significant up-regulation under both salt and osmotic stress.

Three nucleoside diphosphate sugar epimerase genes (CCI6_RS19225, CCI6_RSO8005, CCI6_RSO4525) were upregulated under salt stress, while two nucleoside diphosphate sugar epimerases (CCI6_RS00960, CCI6_RS08005) were up-regulated under osmotic stress. Together, these results suggest that cell wall modifications are involved in the response to salt stress and osmotic stress.

\section{Modulation of membrane composition}

Two genes encoding acyl-acyl carrier protein (ACP) desaturases (CCI6_RS10965, CCI6_RS10965), were upregulated only under salt stress, but not under osmotic stress (Additional file 4). In addition to desaturases, two 
Table 2 qRT-PCR validation of RNA-seq data

\begin{tabular}{|c|c|c|c|c|c|c|}
\hline Locus tag & $\begin{array}{l}\text { qRT-PCR fold change } \\
\text { (salt stress vs control) }\end{array}$ & $\begin{array}{l}\text { RNAseq fold change } \\
\text { (salt stress vs control) }\end{array}$ & $\begin{array}{l}\text { RNAseq vs qRT-PCR } \\
p \text {-value (salt stress) }\end{array}$ & $\begin{array}{l}\text { qRT-PCR fold change } \\
\text { (osmotic stress) }\end{array}$ & $\begin{array}{l}\text { RNAseq fold change } \\
\text { (osmotic stress) }\end{array}$ & $\begin{array}{l}\text { RNAseq vs qRT-PCR } \\
p \text {-value } \\
\text { (osmotic stress) }\end{array}$ \\
\hline CCl6_RS21730a & $2.100^{e}$ & 2.360 & 0.840 & 1.100 & -6.000 & $0.012^{d}$ \\
\hline CCl6_RS17915 & $3.120^{e}$ & 3.240 & 0.960 & $1.820^{\mathrm{e}}$ & 1.980 & 0.870 \\
\hline CCl6_RS19875 & $2.500^{\mathrm{e}}$ & 4.910 & $0.03^{d}$ & $3.011^{e}$ & 11.210 & $0.023^{d}$ \\
\hline CCl6_RS17580 & $3.140^{\mathrm{e}}$ & 3.480 & 0.870 & $2.120^{e}$ & 1.520 & 0.560 \\
\hline CCl6_RS16000 & $1.890^{\mathrm{e}}$ & 2.183 & 0.930 & -0.500 & -1.200 & $0.040^{d}$ \\
\hline CCl6_RS18570 & 1.320 & 1.230 & 0.240 & 1.511 & 1.380 & 0.720 \\
\hline CCl6_RS06495 & 1.400 & 1.500 & 0.450 & -1.310 & -2.020 & 0.220 \\
\hline CCl6_RS02325 & $3.500^{e}$ & 3.840 & 0.830 & $2.800^{e}$ & 3.860 & 0.620 \\
\hline CCl6_RS12340 & -1.300 & -4.000 & $0.023^{d}$ & 1.300 & -9.000 & $0.026^{d}$ \\
\hline CCl6_RS08505 ${ }^{a}$ & $3.230^{e}$ & 3.590 & 0.753 & 1.150 & 1.051 & 0.972 \\
\hline CCl6_RS19950b & 10.021 & 12.061 & 0.778 & 18.231 & 21.585 & 0.812 \\
\hline
\end{tabular}

a Up-regulated genes under salt stress according to RNA-Seq analysis

bUp-regulated genes under both conditions according to RNA-Seq analysis

'Non-differentially expressed genes in the study according to RNA-Seq analysis

Indicates that there is a statistically significant difference between fold change values determined by qPCR and RNA-seq

endicates a statistically significant qRT-PCR fold change value

The fold change values (salt vs control or sucrose vs control) determined from the RNAseq experiment were compared with the fold change values obtained from qRT-PCR experiments. For the qRT-PCR experiments, the $\Delta \Delta C_{t}$ method was used to determine the fold change. The rps $A$ gene was used as the normalizer for all of the qRT-PCR experiments. The fold change values for the RNA-seq experiments are based on two biological replicates whereas the fold change values for the qRT$\mathrm{PCR}$ experiments are based on three biological replicates

other genes [acyl-CoA dehydrogenase (CCI6_RS05135) and $\mathrm{ABC}$-type branched-chain amino acid transport systems, periplasmic component (CCI6_RS10620)] that help determine membrane fluidity were up-regulated under salt stress. Furthermore, salt stress caused the upregulation of ubiE (CCI6_RS17660) gene encoding a multispecies ubiquinone biosynthesis protein. Ubiquinone accumulation has been shown to increase salt tolerance in $E$. coli through mechanical stabilization of the membrane [77]. Taken together, these results suggest that Frankia membrane fluidity was altered by salt stress.

Different osmolytes might be used by the cell depending on the external solute causing the stress

The trehalose synthase gene (CCI6_RS13215) was upregulated under salt stress, while the glutamate synthase
(CCI6_RS10225) and threonine synthase (CCI6_RS08750) genes were up-regulated under osmotic stress. The regulation of intracellular osmolality by the transport or biosynthesis of compatible solutes is believed to be the principal osmoprotection response in bacteria.

\section{Proteomics analysis reveals additional functions that might be involved in salt stress tolerance}

The proteome profile of the salt-tolerant (CcI6) and salt sensitive (CcI3) strains exposed to salt or osmotic stress was examined by the use of 2D SDS-PAGE (Fig. 7). For both strains, prominent changes in protein abundance were readily noticeable under the three different conditions (no stress, salt stress and osmotic stress) and occurred in multiple replicates.

Table 3 Genes unique to the tolerant strain with increased expression under salt stress

\begin{tabular}{|c|c|c|c|c|c|}
\hline \multicolumn{3}{|l|}{ Salt Stress } & \multicolumn{3}{|l|}{ Osmotic Stress } \\
\hline Locus Tag & Protein product & COG category & Locus Tag & Protein product & COG category \\
\hline CCl6_RS13590 & Hypothetical Protein & - & CCl6_RS13590 & Hypothetical Protein & - \\
\hline CCl6_RS22605 & Predicted Zn peptidase & E & CCl6_RS17905 & Acetyltransferase (isoleucine patch superfamily) & $\mathrm{R}$ \\
\hline CCl6_RS17915 & ADP-heptose:LPS heptosyltransferase & M & CCl6_RS13555 & Hypothetical Protein & - \\
\hline CCl6_RS19875 & Hypothetical Protein & - & CCl6_RS15755 & Hypothetical Protein & - \\
\hline CCl6_RS20860 & Hypothetical Protein & - & CCl6_RS21770 & Hypothetical Protein & - \\
\hline CCl6_RS17580 & Hypothetical Protein & - & CCl6_RS13535 & Hypothetical Protein & - \\
\hline CCl6_RS21730 & Hypothetical Protein & - & CCl6_RS02120 & Hypothetical Protein & - \\
\hline
\end{tabular}



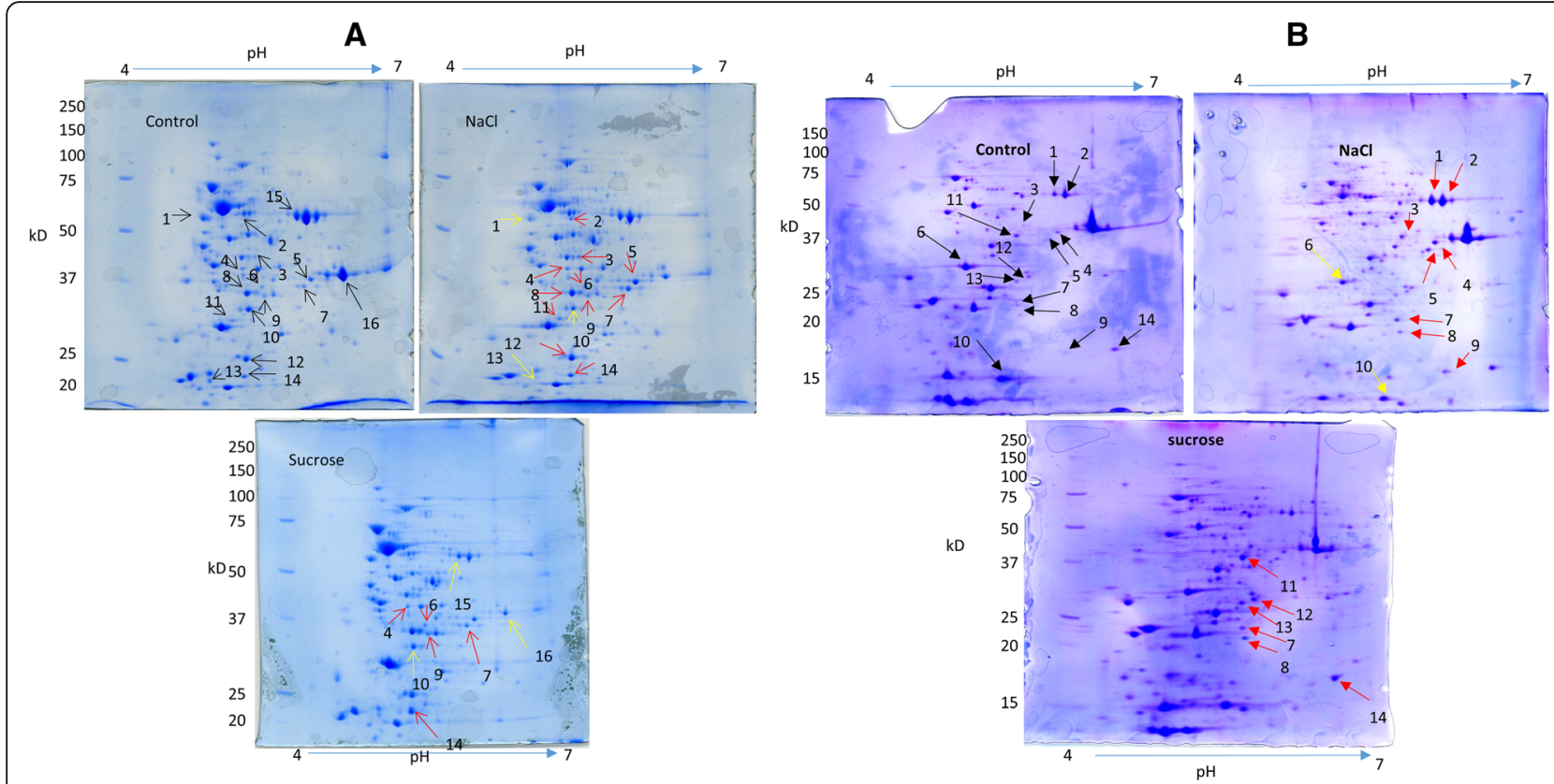

Fig. 7 Two-dimensional polyacrylamide gel electrophoresis (PAGE) analysis of salt stress response. a 2D-gel profile of Frankia sp. strain Ccl6 under control (no stress), $200 \mathrm{mM} \mathrm{NaCl}$, and 200 mM sucrose stress conditions. b 2D-gel profile of Frankia casurainae strain Ccl3 under control (no stress), $200 \mathrm{mM} \mathrm{NaCl}$, and $200 \mathrm{mM}$ sucrose stress conditions. Red arrows indicate that proteins are upregulated relative to the control, while yellow arrows indicate down regulated proteins relative to the control. The corresponding number spots were in-gel digested with trypsin and analyzed by liquid chromatography-mass spectrometry (LC-MS) and LC-MS/MS for protein identification

Differential protein spots were screened and those that showed the most prominent differences between the stress conditions and control gels were targeted for further analysis. For the salt-tolerant strain, 16 differentially expressed spots for all three conditions were analyzed and 19 proteins were identified. For the salt-sensitive strain, 14 differentially expressed spots for all three conditions were analyzed and 8 proteins were identified. For strain CcI6, eleven and three spots were upregulated and downregulated, respectively, under salt stress, while five and three spots were upregulated and downregulated, respectively, under osmotic stress. Out of the 19 saltresponsive strain CcI6 proteins, 18 were assigned into COG functional categories, including energy production and conversion (COG $\mathrm{C}, 4$ proteins), transcription (COG K, 3 proteins), amino acid transport and metabolism (COG E, 2 proteins), post translational modification, protein turn over, chaperon functions (COG O, 2 proteins), carbohydrate transport and metabolism (COG G, 2 proteins) [Table 4].

Among the proteins upregulated in the salt-tolerant strain was a cysteine synthase (CysK) which was identified from a downregulated spot under salt stress (spot no. 10) but had 2.5 times more peptide counts in the $\mathrm{NaCl}$-treated samples. The most abundant protein from the same spot (spot no. 10) was an electron transfer flavoprotein alpha subunit apoprotein (CCI6_RS13315), which, as expected, had a lower peptide count in the
$\mathrm{NaCl}$-treated samples. Several pyridoxal phosphatebinding proteins, including cysteine synthase, are differentially expressed under salt stress in wheat chloroplasts and help in the synthesis of cysteine as a protective measure against toxic ions [78]. Transcriptomics analysis of the salt-tolerant strain (CcI6) revealed that CysK and CysM were upregulated under salt stress. CysK and CysM are pyridoxal phosphate-dependent enzymes. Our proteomics result showed that pyridoxal phosphate synthase yaaD subunit (WP_011435808.1) was upregulated under salt stress in strain CcI6. Another protein from the COG E category that was upregulated under salt stress in strain CcI6 was the glutamine synthetase (WP_011437527.1). Amino acids including proline and glutamine, serve as protective osmolytes under salt stress [25]. The transcriptome analysis of CcI6 showed that glutamine synthetase was upregulated 1.4-fold times, but the change was not statistically significant. A GroEL chaperon (WP_011438752.1) and an ATPdependent $\mathrm{Clp}$ protease proteolytic subunit ClpP (WP_011435649.1) were among the COG O proteins upregulated under salt stress in strain CcI6. In B. subtilis, the synthesis of ClpP protein increases during heat shock, salt and oxidative stress, glucose and oxygen deprivation [79]. Our transcriptome data also showed that the GroEL chaperon (CCI6_RS08745) and ClpP (CCI6_RS22940) are upregulated in salt-stressed CcI6. Another ClpP protein (ClpP_2) [CCI6_RS22535] was 
Table 4 Proteins differentially expressed under stress conditions in strains $\mathrm{Ccl} 6$ and $\mathrm{Ccl} 3$

\begin{tabular}{|c|c|c|c|c|c|c|c|c|}
\hline & SPOT \# & Acc. No & Locus Tag & Protein Name & MW (Da) & $\mathrm{Pl}$ & $\mathrm{NaCl}$ & Sucrose \\
\hline \multicolumn{9}{|c|}{ [C] Energy production and Conversion } \\
\hline \multirow[t]{4}{*}{ Ccl6 } & 10 & $563,313,506$ & CCl6_RS13315 & electron transfer flavoprotein alpha subunit apoprotein & $32,842.70$ & 5.01 & $\downarrow$ & $\downarrow$ \\
\hline & 15 & $563,312,797$ & CCl6_RS16290 & NAD-dependent aldehyde dehydrogenase & $54,456.60$ & 5.40 & N/C & $\downarrow$ \\
\hline & 1 & $563,313,455$ & CCl6_RS13080 & ATP synthase F1 subcomplex beta subunit & $56,735.20$ & 4.56 & $\downarrow$ & $\mathrm{N} / \mathrm{C}$ \\
\hline & 8 & $563,314,955$ & CCl6_RS05745 & malate dehydrogenase (NAD) & $34,399.10$ & 4.96 & $\uparrow$ & $\mathrm{N} / \mathrm{C}$ \\
\hline \multicolumn{9}{|c|}{ [E] Amino acid transport and metabolism } \\
\hline \multirow[t]{2}{*}{$\mathrm{Ccl} 6$} & 2 & $563,312,117$ & CCl6_RS19490 & L-glutamine synthetase & $53,786.20$ & 4.97 & $\uparrow$ & $\mathrm{N} / \mathrm{C}$ \\
\hline & 10 & $563,315,075$ & CCl6_RS05150 & cysteine synthase (CysK) & $32,443.60$ & 4.95 & $\uparrow$ & $\downarrow$ \\
\hline \multicolumn{9}{|c|}{ [G] Carbohydrate transport and metabolism } \\
\hline \multirow[t]{2}{*}{$\mathrm{Ccl} 6$} & 5 & $563,312,326$ & CCl6_RS18410 & glyceraldehyde-3-phosphate dehydrogenase (NAD+) & $35,515.80$ & 5.76 & $\uparrow$ & $\mathrm{N} / \mathrm{C}$ \\
\hline & 7 & $563,314,408$ & CCl6_RS08915 & fructose-bisphosphate aldolase & $36,894.20$ & 5.35 & $\uparrow$ & $\uparrow$ \\
\hline \multirow[t]{3}{*}{$\mathrm{CCl} 3$} & 4 & WP_011436076.1 & FRANCCI3_RS08225 & glyceraldehyde-3-phosphate dehydrogenase (NAD+) & $35,515.80$ & 5.76 & $\uparrow$ & $\mathrm{N} / \mathrm{C}$ \\
\hline & 5 & WP_011438718.1 & FRANCCI3_RS22085 & fructose-bisphosphate aldolase & $36,894.20$ & 5.35 & $\uparrow$ & $\mathrm{N} / \mathrm{C}$ \\
\hline & 11 & WP_011437192.1 & FRANCCI3_RS14110 & aldolase & $38,526.7$ & 5.3 & $\mathrm{~N} / \mathrm{C}$ & $\uparrow$ \\
\hline \multicolumn{9}{|c|}{$[\mathrm{H}]$ Coenzyme metabolism } \\
\hline \multirow[t]{2}{*}{ Ccl6 } & 3 & $563,314,085$ & CCl6_RS10325 & methionine adenosyltransferase & $42,893.60$ & 5.05 & $\uparrow$ & $\mathrm{N} / \mathrm{C}$ \\
\hline & 9 & $563,313,788$ & CCl6_RS11580 & pyridoxal phosphate synthase yaaD subunit & $32,577.80$ & 5.34 & $\uparrow$ & $\uparrow$ \\
\hline $\mathrm{CCl} 3$ & 9 & WP_011437570.1 & FRANCCI3_RS16030 & 6,7-dimethyl-8-ribityllumazine synthase & $16,137.8$ & 5.5 & $\uparrow$ & $\mathrm{N} / \mathrm{C}$ \\
\hline \multicolumn{9}{|c|}{ [1] Lipid transport and metabolism } \\
\hline $\mathrm{Ccl} 3$ & 3 & WP_011438063 & & acetyl-CoA acetyltransferase & $39,714.5$ & 5.3 & $\uparrow$ & $\mathrm{N} / \mathrm{C}$ \\
\hline \multicolumn{9}{|c|}{ [J] Translation } \\
\hline $\mathrm{Ccl} 3$ & 8 & WP_011437955.1 & FRANCCI3_RS18065 & ribosome-recycling factor & $20,830.7$ & 5.4 & $\uparrow$ & $\uparrow$ \\
\hline \multicolumn{9}{|c|}{$[\mathrm{K}]$ Transcription } \\
\hline \multirow[t]{3}{*}{ Ccl6 } & 3 & $563,314,632$ & CCl6_RS07960 & $\begin{array}{l}\text { DNA-directed RNA polymerase, sigma subunit } \\
\text { (sigma70/sigma32) }\end{array}$ & $44,399.30$ & 5.10 & $\uparrow$ & $\mathrm{N} / \mathrm{C}$ \\
\hline & 12 & $563,312,999$ & CCl6_RS15595 & RNA polymerase, sigma 24 factor & $29,218.90$ & 5.49 & $\downarrow$ & $\mathrm{N} / \mathrm{C}$ \\
\hline & 13 & $563,314,238$ & CCl6_RS09105 & Transcriptional regulator Crp/Fnr & $51,393.10$ & 4.97 & $\downarrow$ & $\mathrm{N} / \mathrm{C}$ \\
\hline $\mathrm{Ccl} 3$ & 14 & WP_011437589.1 & FRANCCI3_RS16125 & XRE family transcriptional regulator & $17,993.4$ & 5.7 & $\uparrow$ & $\mathrm{N} / \mathrm{C}$ \\
\hline \multicolumn{9}{|c|}{ [M] Cell wall/membrane/envelop biogenesis } \\
\hline \multirow[t]{2}{*}{ Ccl6 } & 6 & $563,313,716$ & CCl6_RS12035 & UDP-glucose pyrophosphorylase & $34,856.10$ & 5.04 & $\uparrow$ & $\uparrow$ \\
\hline & 11 & $563,315,562$ & CCl6_RS03270 & $\begin{array}{l}\text { Nucleoside-diphosphate-sugar pyrophosphorylase } \\
\text { family protein }\end{array}$ & $31,492.90$ & 4.92 & $\uparrow$ & $\mathrm{N} / \mathrm{C}$ \\
\hline \multicolumn{9}{|c|}{ [O] Post-translational modification, protein turnover, chaperone functions } \\
\hline \multirow[t]{2}{*}{$\mathrm{Ccl} 6$} & 2 & $563,314,374$ & CCl6_RS08745 & chaperonin GroEL & $56,735.20$ & 4.72 & $\uparrow$ & $\mathrm{N} / \mathrm{C}$ \\
\hline & 14 & $563,311,297$ & CCl6_RS22940 & ATP-dependent Clp protease proteolytic subunit ClpP & $23,039.30$ & 4.79 & $\uparrow$ & $\uparrow$ \\
\hline \multicolumn{9}{|c|}{ [R] General Functional Prediction only } \\
\hline $\mathrm{Ccl} 6$ & 16 & $563,312,796$ & CCl6_RS16285 & Zn-dependent alcohol dehydrogenase & $35,062.50$ & 5.65 & $\mathrm{~N} / \mathrm{C}$ & $\downarrow$ \\
\hline $\mathrm{Ccl} 3$ & 7 & WP_011434678.1 & FRANCCI3_RS01050 & FMN reductase & $21,222.1$ & 5.3 & $\uparrow$ & $\uparrow$ \\
\hline \multicolumn{9}{|c|}{ Not assigned to COG categories } \\
\hline \multirow[t]{2}{*}{$\mathrm{Ccl} 6$} & 4 & $563,313,603$ & CCl6_RS12255 & Nitroreductase & $37,351.40$ & 4.90 & $\uparrow$ & $\uparrow$ \\
\hline & 12 & $563,313,563$ & CCl6_RS12810 & proteasome endopeptidase complex & $28,833.80$ & 5.11 & $\uparrow$ & $\mathrm{N} / \mathrm{C}$ \\
\hline
\end{tabular}

The identified proteins were classified by COG functional categories. More than one protein per spot has been identified for some spots. Upregulated proteins are shown by the upward pointing arrow $(\uparrow)$ whereas downregulated proteins are shown by the downward pointing arrow $(\downarrow)$. No change (N/C) indicates that a spot was not picked for that particular condition because it showed similar intensity as the control 
upregulated under osmotic stress in strain CcI6 based on the RNA-seq data, but this protein was not identified in our proteomics data. GroEL is one of the heat shock proteins and shows upregulation under salt stress in Lactococcus lactis, suggesting there is overlap between salt and heat stress responses [80]. In strain CcI6, among differentially expressed proteins identified in the COG $\mathrm{K}$ functional category, a DNA-directed RNA polymerase sigma subunit (WP_023840564.1) was upregulated under salt stress. The transcriptome analysis did not reveal upregulation of the sigma factor, suggesting posttranscriptional regulation of the transcript. An extracytoplasmic function (ECF) family RNA polymerase, sigma subunit (WP_035729933.1) was downregulated under salt stress in strain CcI6. Our transcriptome analysis also showed downregulation of the ECF family sigma factor. The ECF sigma factors are small divergent group of regulatory proteins that control the transcription of genes associated with response to extracytoplasmic stress conditions and some aspect of the cell surface or transport [81]. Two proteins, glyceraldehyde-3phosphate dehydrogenase and fructose-bisphosphate aldolase, belonging to the carbohydrate transport and metabolism functional category (COG G), were upregulated under salt stress in strain CcI6. Overexpression of glyceraldehyde-3-phosphate dehydrogenase in rice plants improved salt tolerance [82]. Similarly, the overexpression of fructose-bisphosphate aldolase in Brassica napus led to increased salt stress tolerance [83]. Another salttolerant strain, Allo2, showed similar changes in the proteome prolife as strain CcI6 under salt and osmotic stress conditions (Additional files 5,6).

For strain $\mathrm{CcI} 3$, seven and three proteins were identified from upregulated spots under salt and osmotic stress conditions, respectively. All of the proteins upregulated under osmotic stress were also upregulated under salt stress. CcI3 proteins upregulated under salt stress include glyceraldehyde-3-phosphate dehydrogenase (COG G), fructose-bisphosphate aldolase (GOC G), ribosome-recycling factor (COG J), aldolase (COG G), XRE family transcriptional regulator (COG K), 6,7-dimethyl-8-ribityllumazine synthase (COG $\mathrm{H}$ ), FMN reductase (COG R), and acetyl-CoA acetyltransferase (COG I). Two of the proteins upregulated under salt stress in CcI3, glyceraldehyde-3-phosphate dehydrogenase and fructose-bisphosphate aldolase, show a similar pattern of upregulation in all three strains $(\mathrm{CcI} 3, \mathrm{CcI} 6$, and Allo2).

\section{Discussion}

Salt tolerance mechanisms depend on the availability of nitrogen

The salt tolerance levels for strains Allo2 and CcI6 (1000 mM) were comparable to the 500-1500 mM NaCl tolerance reported for moderately halophilic grampositive bacteria [84]. Strain CcI3 exhibited about half of the salt tolerance level $(475 \mathrm{mM})$ of the two salt-tolerant strains, only slightly less than the level $(528 \mathrm{mM})$ for Rhizobium meliloti, a salt-tolerant rhizobium species [85]. Non-Casuarina isolates in general, and strain EuI1C in particular, outperformed the Casuarina isolates under osmotic stress imposed by sucrose. The results suggest that Casuarina isolates have developed a mechanism that specifically copes with the toxic effects of $\mathrm{Na}$ ${ }^{+}$and $\mathrm{Cl}^{-}$ions. This situation also occurs with Calobacter [86]. The observed difference in salt tolerance levels between salt-tolerant and salt-sensitive Casuarina isolates dissipated under nitrogen-deficient $\left(\mathrm{N}_{2}\right)$ conditions, suggests that salt stress response is reliant on ample supply of nitrogen sources. The result is expected as many of the osmotic adjustments that take place -from protective osmolyte synthesis to cell envelope remodelingrely heavily on nitrogen containing compounds. Under nitrogen-deficient conditions, survival of Frankia is dependent on the activities of the nitrogenase enzyme, which reduces atmospheric dinitrogen into ammonia. For all Casuarina isolates, both nitrogenase activity and vesicle number per milligram of protein decreased with increasing levels of salinity, causing a compound effect on nitrogen fixation. The fact that nitrogenase activity was affected more severely than overall growth with increasing concentrations of $\mathrm{NaCl}$, and the fact that external nitrogen supply dramatically improves the salt tolerance of strains suggests that the ability to maintain nitrogen fixation under salt-stress is important for salt stress tolerance.

\section{All of the Casuarina isolates are the same species}

Based on a cutoff value of $95 \%$ used for species delineation [61], the ANI values (>99\%) and the AAI values (>98\%) observed between any two pairs of Casuarina isolates indicate that all Casuarina isolates belong to the same species, Frankia casuarinae, and are distinct from the closely related cluster Ia isolate Frankia alni strain ACN14a. The concatenated phylogenetic affiliation of 394 maximum-parsimony trees based on amino acid sequences also reveals that Casuarina isolates group together and are distinct from the closely related cluster Ia isolate strain ACN14a. The two highly salt-tolerant strains (Allo2 and CcI6) were the closest two strain having the lowest genome- to- genome distance, the highest ANI, and AAI. It is tempting to suggest that the salt tolerance mechanism of the two strains is shared. This idea was confirmed by the proteomic analysis of the two strains under salt stress which revealed a similar pattern of differentially expressed proteins. A shared salttolerance mechanism with a common origin for the two strains is further supported by pan-genome analysis of 
the Casuarina isolates which revealed hundreds of single copy genes that are exclusively shared between the two salt-tolerant strains. Transcriptome analysis revealed some of these tolerant strain-specific genes are responsive to salt and osmotic stress.

\section{Differences between RNA-seq and proteome results}

After identifying genetic differences between the salttolerant and the salt-sensitive strains, we proceeded with transcriptomics and proteomics to determine if the genetic difference includes genes that are responsive to salt and osmotic stress. Because of factors such as half-lives and post transcription machinery, the correlation between mRNA and protein expressions can be low. Therefore, joint analysis of transcriptomic and proteomic data can provide useful insight that is otherwise impossible to obtain from individual analysis of mRNA or protein expressions [87]. Our results suggest that both transcriptional and post-transcriptional controls are involved in the regulation of genes under hyper-osmotic stress in strain CcI6.

\section{Altered expression of regulatory proteins}

Alternative sigma factors and transcription regulators are involved in controlling expression of stressresponsive genes and help to create flexibility in the adaptation of cells to environmental stress [88]. Several genes encoding transcriptional factors from the GntR, TetR, LysR, and Crp/Fnr families were up-regulated under salt stress. Similarly, several genes encoding transcriptional regulators from the Crp/Fnr and LuxR families were up-regulated under osmotic challenge. Proteomics analysis did not reveal any transcriptional factors, probably because they are low abundance proteins that fall below the detection range. Under salt stress, only one sigma factor (CcI6_RS19210), which has a SigF domain, was up-regulated in Frankia sp. strain CcI6. A similar pattern of up-regulation under salt stress was also found in strains $\mathrm{CcI} 3$ and $\mathrm{CeD}$ (unpublished data). The sigF regulon in Mycobacterium smegmatis mediates stationary phase adaptation and general stress response [89]. Mycobacterium smegmatis SigF was suggested to regulate the biosynthesis of the osmoprotectant trehalose and an uptake system for osmoregulatory compounds. Our transcriptome results showed an upregulation of trehalose synthase under salt stress. SigF has also been implicated in the direct control of gene expression for regulatory proteins SigH3, PhoP, WhiB1, and WhiB4. Under salt stress, only one sigma factor, an ECF sigma factor (CCI6_RS15595), was down-regulated under salt stress in Frankia strain CcI6. This result is in contrast with the reported auto-upregulation of ECF sigma factors in response to extracytoplasmic stress conditions, including salt stress [90]. ECF sigma factors recognize promoter elements with an 'AAC' motif in the -35 region and are usually co-transcribed with a transmembrane anti-sigma factor with an extracytoplasmic sensory domain and an intracellular inhibitory domain. In the strain CcI6 genome, the down-regulated ECF sigma factor lies upstream of a mycothiol system antisigma- $\mathrm{R}$ factor, suggesting that the ECF sigma factor is a homologue of sigma R. In Streptomyces coelicolor A3(2), sigma $\mathrm{R}$ is regulated by the cognate anti-sigma- $\mathrm{R}$ factor (RsrA), which loses affinity for sigma $\mathrm{R}$ following oxidative stress that introduces intramolecular disulphide bond formation in RsrA [91]. ECF sigma factors are regulated at the transcriptional, translational, and posttranslational levels [90]. The transcriptional control of ECF factors can involve a hierarchical regulatory cascade of sigma factors. The most important regulation of ECF sigma factors involves the reversible binding of the sigma factor to an anti-sigma factor, holding it in an inactive complex as long as the cognate environmental stimulus is absent. Changes in environmental conditions sensed by the anti-sigma factor lead to release of the sigma factor, which will subsequently bind to the RNA polymerase core enzyme and initiate transcription [92]. Analysis of the proteome data, but not the transcriptome, showed that a $\sigma^{32}$ factor involved in the cytoplasmic heat shock response is up-regulated under salt stress. This agrees with the result from the transcriptome analysis where the small heat-shock protein Hsp20 was up-regulated under salt stress.

\section{Potential mechanisms of salt tolerance}

All Casuarina isolates had the same set of classical genes involved in salt and osmotic stress tolerance, suggesting the observed difference in tolerance was due to previously unknown or less characterized mechanisms. All Casuarina isolates lacked the BCCT family transporters, which are present in the closely related strain ACN14a, and lacked the ability to synthesize or acquire glycine betaine (or the precursor choline) from the environment. Strains CcI6 and Allo2 manifest high salt tolerance in a minimal growth medium confirming the idea that the ability to acquire glycine betaine from the environment is not a key factor in the salt tolerance of Casuarina isolates.

The majority of the hundreds of tolerant-strainspecific genes code for hypothetical proteins, suggesting novel mechanisms responsible for the tolerance. The remaining tolerant-strain-specific genes include those involved in replication, recombination and repair; and cell wall/membrane biogenesis. This would indicate that the ability to maintain the integrity of the genetic material, the replication process, and the cell envelope are all important for salt tolerance. The presence of unique genes in the salt-tolerant strains that are involved in cell wall 
and membrane biogenesis suggests that pre-existing differences in membrane/cell wall composition and structure might be contributing factors to the observed difference in salt tolerance levels between salt-sensitive and salt-tolerant strains.

Among the tolerant-strain-specific genes, only a small fraction was responsive to both salt and osmotic stress. The majority of those genes encoded hypothetical proteins, suggesting that novel mechanisms are responsible for the observed difference in salt-tolerance between strain $\mathrm{CcI} 3$ and the two salt-tolerant strains (CcI6 and Allo2). A zinc peptidase gene was among the salttolerant strain-specific genes that were responsive to salt stress. Transcriptome analysis of salt-tolerant and saltsensitive varieties of rice had a zinc peptidase gene as one of the 50 top responsive genes [74]. The zinc peptidase gene up-regulated in strain CcI6 under salt stress has a DNA binding domain, suggesting it might play a role in response regulation. Salt stress and the accompanying oxidative stress leads to structural changes that compromise the function of proteins. Misfolded and aggregated proteins are degraded by proteases. Proteases are increasingly being associated with salt tolerance and sensitivity to abiotic stress [93]. None of the gene products unique to the salt-tolerant strains were identified from our proteomics analysis probably because they were present in amounts below the detection threshold.

From transcriptome analysis of strain CcI6, hundreds of genes were differentially expressed under salt and osmotic stress conditions. There was a clear overlap between salt and osmotic stress responses, but most of the responses were condition-specific. This result partly explains why there is a huge disparity between the salt and osmotic stress tolerance levels among the Casuarina isolates. The mechanism by which each functional category contributes towards salt-tolerance is discussed in the following paragraphs.

\section{Alterations of the cell envelope}

The composition of the cell envelope plays an important role in osmo-adaptation [94]. Cell envelope-related changes triggered by salt stress include alterations in the structure and composition of the peptidoglycan layer [95], and changes in membrane and/or periplasmic protein composition, lipid composition, periplasmic glucan levels, and capsular polysaccharide biosynthesis [96]. These modifications to the cell wall under salt stress create a diffusion barrier to reduce the influx of inorganic ions [97]. Salt-stress induced alterations of the peptidoglycan layer involve several enzymes including glycolyl transferases, polysaccharide deacetylases and sugar epimerases. Our transcriptome data showed that several glycosyl transferases and nucleocide diphosphate sugar epimerases involved in cell wall/membrane biogenesis were up-regulated under both salt and osmotic stress conditions indicating that the cell envelope was being altered. However, different glycosyl transferases and nucleocide diphosphate sugar epimerases were up-regulated under each condition suggesting that these cell envelope changes were different under salt or osmotic stress.

\section{Changes in membrane fluidity}

Regulation of membrane fluidity in response to osmotic stress is also an important aspect of cell envelope remodeling during salt stress [98]. Regulation of membrane fluidity mainly involves changes in the fatty acid composition of the membrane by varying the length of acyl chains, number of double bonds or branching of acyl chains by methyl groups [99]. Our transcriptome results show an up-regulation of ACP desaturases (CCI6_RS10965, CCI6_RS10965) genes only under salt stress, but not under osmotic stress. ACP desaturases catalyze the conversion of saturated fatty acids into unsaturated fatty acids by the introduction of at least one double bond. This result would indicate that the membrane has elevated levels of unsaturated fatty acids indicating an increased fluidity.

Salt stress also caused the up-regulation of $u b i E$ (CCI6_RS17660) encoding a multispecies ubiquinone biosynthesis protein. In $E$. coli, ubiquinone accumulation increases salt tolerance through mechanical stabilization of the membrane [77]. Taken together, these results suggest that Frankia membrane fluidity was altered by salt stress.

\section{Compatible solutes}

The accumulation of compatible solutes is one of the major physiological coping mechanisms employed by cells exposed to a hyper osmotic pressure. Among the commonly used compatible solutes are the sugar trehalose and various amino acids [30]. Genes coding for enzymes involved in trehalose synthesis were up-regulated under salt stress while genes coding for enzymes involved in the synthesis of threonine and glutamate were up-regulated under osmotic stress. Trehalose serves as a universal stress molecule and plays a role in the cellular adaptation to high osmolarity, heat, oxidation, desiccation and freezing [100]. The role of osmolytes transcends maintaining cell turgor by increasing intracellular osmolality. Molecular dynamics studies have demonstrated an interaction between the osmolyte trehalose and the membrane lipid head groups, although the observed resistance of membranes to strong osmotic stress could not fully be ascribed to the interaction [101]. The increased expression of the trehalose synthase gene only under chronic salt stress and the increase in expression of the threonine and glutamate synthase genes only under osmotic stress (induced by sucrose) suggests that the preferred protective osmolyte depends on the external solute causing the stress. 


\section{Conclusions}

Comparative genomics, transcriptome, and proteome analyses of Frankia strains isolated from Casuarina trees revealed that salt stress response involves differential expression of a myriad of genes from various functional categories. Most of the genes specific to the salt-tolerant strains coded for hypothetical proteins and many saltstress responsive genes from the transcriptome and proteome profiles coded for hypothetical proteins. This line of evidence suggests a role for these hypothetical proteins in salt-stress response and may represent a previously uncharacterized novel mechanism(s) for salt tolerance. The development of genetic tools for the mutational analysis of some of the candidate genes coupled with biochemical and physiological analysis could yield insights on these novel mechanisms of salt tolerance.

\section{Additional files}

Additional file 1: Table S1. List of primers used for $q R T-P C R$ validation of RNA-seq data. (DOCX $12 \mathrm{~kb}$ )

Additional file 2: The effect of salt stress on the vesicle formation and nitrogenase activity by salt-tolerant and salt-sensitive Casuarina isolates. Cultures were grown under nitrogen-deficient conditions with various degrees of salt or osmotic stress. Panel (A) shows vesicle production. Panel (B) show nitrogenase activity expressed on a per-vesicle basis. (PPTX 64 kb)

Additional file 3: List of 153 single-copy genes shared between Frankia sp. strain Ccl6 and Allo2, but are absent in Frankia sp. strain Ccl3 and $\mathrm{CeD}$, as determined by the modified lerat program. List of 132 genes present in Frankia sp. strain Allo2 and Ccl6, but are absent in other Casuarina associated Frankia sp. strains (Ccl3, CeD, Thr, and BMG5.23), as determined by OrthoVenn. (XLSX $13 \mathrm{~kb})$

Additional file 4: Frankia sp. strain Ccl6 genes differentially expressed under salt and osmotic stress based on RNAseq analysis. (XLSX $87 \mathrm{~kb}$ )

Additional file 5: Two-dimensional polyacrylamide gel electrophoresis (PAGE) analysis of Frankia sp. strain Allo2 under control (no stress) conditions (A), $200 \mathrm{mM} \mathrm{NaCl}$ (B), and $200 \mathrm{mM}$ sucrose (C). Red arrows indicate that proteins are up-regulated relative to the control, while yellow arrows indicate down regulated proteins relative to the control. The corresponding number spots were in-gel digested with trypsin and analyzed by liquid chromatography-mass spectrometry (LC-MS) and LC-MS/ MS for protein identification. (PPTX $653 \mathrm{~kb}$ )

Additional file 6: Table S3. Frankia sp. strain Allo2 proteins differentially expressed under stress conditions. The identified proteins were classified by COG functional categories. Up-regulated proteins are shown by the upward pointing arrow $(\uparrow)$ whereas down-regulated proteins are shown by the downward pointing arrow $(\downarrow)$. No change (N/ C) indicates that a spot was not picked for that particular condition because it showed similar intensity as the control. (DOCX 15 kb)

\section{Abbreviations}

AAl: Average amino acid identity; ACN: Acetonitrile; ANI: Average nucleotide identity; CDS: Coding DNA sequence; CID: Low-energy collision-induced dissociation; COG: Cluster of Orthologous Groups of protein; DDH: DNA-DNA hybridization; Ddl: D-alanine ligase; DTT: Dithiothreitol; GGP: Geranylgeranyl pyrophosphate; GGPS: Geranylgeranyl pyrophosphate synthase; IAA: lodoacetamide; IEF: Isoelectric focusing; IPG: Immobiline pH gradient; LC-MS: Liquid chromatography - mass spectrometry; MIC: Minimum inhibitory concentration; MTC: Maximum tolerable concentration; NAGGN: Nacetylglutaminylglutamine amide; OtsA: Trehalose-6-phosphate synthase; OtsB: Trehalose-6-phosphate phosphatase; qRT-PCR: Quantitative reverse transcription PCR; RIN: RNA integrity number; ROS: Reactive oxygen species; SDS: Sodium dodecyl sulfate; TCA: Trichloroacetic acid

\section{Acknowledgements}

This is Scientific Contribution Number 2720.

\section{Funding}

Partial funding was provided by the New Hampshire Agricultural Experiment Station (LST). This project (LST) was supported in part by USDA National Institute of Food and Agriculture Hatch 022821, and the College of Life Sciences and Agriculture (COLSA) at the University of New Hampshire, Durham, $\mathrm{NH}$. MN was supported by the MERS (Ministère de l'Enseignement Supérieur et de la Recherche du Sénégal, national grant), the WFS (Word Federation of Scientists, research allowance) and an ARTS (Allocation de Recherche pour une Thèse au Sud), PhD grant from the IRD (Institut de Recherche pour le Développement). These funding sources had no role in the design, collection, analysis, and interpretation of the data and in writing the manuscript.

\section{Availability of data and materials}

The datasets generated during the current study are available in the NCBI Gene Expression Omnibus database (http://www.ncbi.nlm.nih.gov) under accession number (GSE95217). Other data sets are included as supplemental materials.

\section{Authors' contributions}

$\mathrm{RO}$ carried out the experiments, analyzed the data, interpreted the results, created the figure, and drafted of the manuscript. LST guided data acquisition methodology and critically revised the intellectual content of the manuscript for submission. MN conducted some experiments, interpreted the results, and improved the manuscript. FC, SM, MOS, AC interpreted the results and improved the manuscript. All authors have read and approved the final manuscript.

\section{Ethics approval and consent to participate}

Not applicable. This study did not involve humans, animals or plants in any manner.

\section{Consent for publication}

Not applicable.

\section{Competing interests}

The authors declare that they have no competing interests.

\section{Publisher's Note}

Springer Nature remains neutral with regard to jurisdictional claims in published maps and institutional affiliations.

\section{Author details}

${ }^{1}$ Department of Molecular, Cellular and Biomedical Sciences, University of New Hampshire, 46 College Rd, Durham, NH 03824-2617, USA. ²aboratoire Mixte International Adaptation des Plantes et microorganismes associés aux Stress Environnementaux, Centre de Recherche de Bel-Air, Dakar, Sénégal. ${ }^{3}$ Laboratoire Campus de Biotechnologies Végétales, Département de Végétale, Faculté des Sciences et Techniques, Université Cheikh Anta Diop, Dakar, Sénégal. ${ }^{4}$ Laboratoire Commun de Microbiologie Institut de Recherche pour le Développement/Institut Sénégalais de Recherches Agricoles/Université Cheikh Anta Diop, Centre de Recherche de Bel-Air, Dakar, Sénégal. ${ }^{5}$ Faculty of Science, Suez Canal University, Ismalia, Egypt. ${ }^{6}$ UMR DIADE, Institut de Recherche pour le Développement, Montpellier, France.

Received: 27 April 2017 Accepted: 11 August 2017

Published online: 18 August 2017

\section{References}

1. Shrivastava P, Kumar R. Soil salinity. A serious environmental issue and plant growth promoting bacteria as one of the tools for its alleviation. Saudi Journal of Biological Sciences. 2015;22:123-31.

2. Jamil A, Riaz S, Ashraf M, Foolad MR. Gene Expression Profiling of Plants under Salt Stress. Crit Rev Plant Sci. 2011;30:435-58.

3. Haffez FY, Hameed S, Malik KA. Frankia and Rhizobium strains as inoculum for fast growing trees in saline environment. Pak J Bot. 1999;31:173-82.

4. Tani C, Sasakawa H. Salt tolerance of Casuarina equisetifolia and Frankia Ceq1 strain isolated from the root nodules of C. equisetifolia. Soil Science and Plant Nutrition. 2003;49:215-22. 
5. El-Lakany MH, Luard EJ. Comparative salt tolerance of selected Casuarina species. Australian Forest Research. 1982;13:11-20.

6. Girgis MGZ, Ishac YZ, Diem HG, Dommergues YR. Selection of salt-tolerant Casuarina glauca and Frankia. Acta Oecol. 1992;13:443-51.

7. Mailly D, Ndiaye P, Margolis HA, Pineau M. Fixation des dunes et reboisement avec le filao (Casuarina equisetifolia) dans la zone du littoral nord du Sénégal. For Chron. 1994;70:282-90

8. Doran JC, Hall N. Notes on fifteen Australian Casuarina species. In: Midgeiey SJ, Tumbuil JW, Johnson RD, editors. Casuarina ecology, management and utilization: proceedings of an international workshop, Canberra, Australia. 17-21 August 1981. Melbourne: CSIRO; 1983. p. 19-52.

9. Baker DD, Schwintzer CR. Introduction. In: Schwintzer CR, Tjepkema JD, editors. The biology of Frankia and actinorhizal plants. San Diego: Academic Press; 1990. p. 3-11.

10. Baker DD, Mullin BC. Actinorhizal symbioses. In: Stacey G, Burris RH, Evans HJ, editors. Biological nitrogen fixation. New York: Chapman and Hall; 1992. p. 259-92.

11. Echbab H, Arahou M, Ducousso M, Nourissier-Mountou S, Duponnois R, Lahlou $\mathrm{H}$, et al. Successful nodulation of Casuarina by Frankia in axenic conditions. J Appl Microbiol. 2007;103:1728-37.

12. Schwencke J, Carú M. Advances in Actinorhizal Symbiosis: Host PlantFrankia Interactions, Biology, and Applications in Arid Land Reclamation. A Review. Arid Land Res Manag. 2001;15:285-327.

13. Dawson JO, Gibson AH. Sensitivity of selected Frankia isolates from Casuarina, Allocasuarina and North American host plants to sodium chloride. Physiol Plant. 1987;70:272-8.

14. Oshone R, Mansour SR, Tisa LS. Effect of salt stress on the physiology of Frankia sp strain Ccl6. J Biosci. 2013;38:699-702.

15. Ghodhbane-Gtari F, Hurst SG, Oshone R, Morris K, Abebe-Akele F, Thomas WK, et al. Draft Genome Sequence of Frankia sp. Strain BMG5.23, a Salt-Tolerant Nitrogen-Fixing Actinobacterium Isolated from the Root Nodules of Casuarina glauca grown in Tunisia. Genome Announcements. 2014:2:e00520-14.

16. Hurst SG, Oshone R, Ghodhbane-Gtari F, Morris K, Abebe-Akele F, Thomas WK, et al. Draft Genome Sequence of Frankia sp. strain Thr, a Nitrogen-Fixing Actinobacterium Isolated from the Root Nodules of Casuarina cunninghamiana grown in Egypt. Genome Announcements. 2014;2:e00493-14.

17. Mansour SR, Oshone R, Hurst SG, Morris K, Thomas WK, Tisa LS. Draft Genome Sequence of Frankia sp. strain Ccl6, a Salt-Tolerant Nitrogen-Fixing Actinobacterium Isolated from the Root Nodule of Casuarina cunninghamiana. Genome Announcements. 2014;2:e01205-13.

18. Ngom M, Oshone R, Hurst SG IV, Abebe-Akele F, Simpson S, Morris K, Sy MO, Champion A, Thomas WK, Tisa LS. Permanent draft genome sequence for Frankia sp. strain CeD, a nitrogen-fixing actinobacterium isolated from the root nodules of Casuarina equistifolia grown in Senegal. Genome Announcements. 2016;4:e00265-16.

19. D'Angelo T, Oshone R, Abebe-Akele F, Simpson S, Morris K, Thomas WK, et al. Permanent Draft Genome Sequence of Frankia sp. Strain BR, a NitrogenFixing Actinobacterium Isolated from the Root Nodules of Casuarina equisetifolia. Genome Announcements. 2016:4:e01000-16.

20. Normand P, Lapierre P, Tisa LS, Gogarten JP, Alloisio N, Bagnarol E, et al. Genome characteristics of facultatively symbiotic Frankia sp. strains reflect host range and host plant biogeography. Genome Res. 2006;17:7-15.

21. Oshone R, Ngom M, Abebe-Akele F, Simpson S, Morris K, Sy MO, et al. Permanent Draft Genome Sequence of Frankia sp. Strain Allo2, a SaltTolerant Nitrogen-Fixing Actinobacterium Isolated from the Root Nodules of Allocasuarina. Genome Announcements. 2016;4:e00388-16.

22. Tisa LS, Oshone R, Sarkar I, Ktari A, Sen A, Gtari M. Genomic approaches toward understanding the actinorhizal symbiosis: an update on the status of the Frankia genomes. Symbiosis. 2016;70:5-16.

23. Brown A. Microbial water stress. Bacteriol Rev. 1976;40:803-46.

24. Waditee R, Hibino T, Nakamura T, Incharoensakdi A, Takabe T. Overexpression of a $\mathrm{Na} / \mathrm{H}$ antiporter confers salt tolerance on a freshwater cyanobacterium, making it capable of growth in sea water. Proc Natl Acad Sci. 2002;99:4109-14.

25. Shamseldin A, Nyalwidhe J, Werner D. A Proteomic Approach Towards the Analysis of Salt Tolerance in Rhizobium etli and Sinorhizobium meliloti Strains. Curr Microbiol. 2006;52:333-9.

26. Romantsov T, Guan Z, Wood JM. Cardiolipin and the osmotic stress responses of bacteria. Biochim Biophys Acta Biomembr. 2009;1788:2092-100.

27. Brígido C, Alexandre A, Oliveira S. Transcriptional analysis of major chaperone genes in salt-tolerant and salt-sensitive mesorhizobia. Microbiol Res. 2012;167:623-9.
28. Ventosa A, Nieto JJ, Oren A. Biology of moderately halophilic aerobic bacteria. Microbiol Mol Biol Rev. 1998;62:504-44.

29. Smith LT, Smith GM. An osmoregulated dipeptide in stressed Rhizobium meliloti. J Bacteriol. 1989;171:4714-7.

30. TeChien C, Maundu J, Cavaness J, Dandurand LM, Orser CS. Characterization of salt-tolerant and salt-sensitive mutants of Rhizobium leguminosarum biovar viciae strain C1204b. FEMS Microbiol Lett. 1992;90:135-40.

31. Tsuzuki M, Moskvin OV, Kuribayashi M, Sato K, Retamal S, Abo M, et al. Salt Stress-Induced Changes in the Transcriptome, Compatible Solutes, and Membrane Lipids in the Facultatively Phototrophic Bacterium Rhodobacter sphaeroides. Appl Environ Microbiol. 2011;77:7551-9.

32. Peddie B. Relationship between osmoprotection and the structure and intracellular accumulation of betaines by Escherichia coli. FEMS Microbiol Lett. 1994;120:125-31.

33. Ko R, Smith LT, Smith GM. Glycine betaine confers enhanced osmotolerance and cryotolerance on Listeria monocytogenes. J Bacteriol. 1994;176:426-31.

34. Sleator R. Bacterial osmoadaptation: the role of osmolytes in bacterial stress and virulence. FEMS Microbiol Rev. 2001;25

35. Miller G, Suzuki N, Ciftci-Yilmaz S, Mittler R. Reactive oxygen species homeostasis and signalling during drought and salinity stresses. Plant Cell Environ. 2010;33:453-67.

36. Apel K, Hirt H. Reactive Oxygen Species: Metabolism, Oxidative Stress, and Signal Transduction. Annu Rev Plant Biol. 2004;55:373-99.

37. Singh S, Brocker C, Koppaka V, Chen Y, Jackson BC, Matsumoto A, et al. Aldehyde dehydrogenases in cellular responses to oxidative/electrophilic stress. Free Radic Biol Med. 2013;56:89-101.

38. Bajji M, Kinet JM, Lutts S. The use of the electrolyte leakage method for assessing cell membrane stability as a water stress tolerance test in durum wheat. Plant Growth Regul. 2001;36:61-70.

39. Turk M, Méjanelle L, Šentjurc M, Grimalt JO, Gunde-Cimerman N, Plemenitaš A. Salt-induced changes in lipid composition and membrane fluidity of halophilic yeast-like melanized fungi. Extremophiles. 2003;8:53-61.

40. Tasaka Y, Gombos Z, Nishiyama Y, Mohanty P, Ohba T, Ohki K, Murata N. Targeted mutagenesis of acyl-lipid desaturases in Synechocystis: evidence for the important roles of polyunsaturated membrane lipids in growth, respiration and photosynthesis. EMBO J. 1996;15:6416-25.

41. Srivastava A, Singh SS, Mishra AK. Modulation in fatty acid composition influences salinity stress tolerance in Frankia strains. Ann Microbiol. 2013;64:1315-23.

42. Mansour SR, Moussa LAA. Role of Gamma-radiation on spore germination and infectivity of Frankia strains Cel523 and Ccl6 isolated from Egyptian Casuarina. Isotope and Radiation Research. 2005;37:1023-38.

43. Girgis MGZ, Schwencke J. Differentiation of Frankia strains by their electrophoretic patterns of intracellular esterases and aminopeptidases. J Gen Microbiol. 1993;139:2225-32.

44. Girgis MGZ, Ishac YZ, El-Haddad M, Saleh EA, Diem HG, Dommergues YR. First report on isolation and culture of effective Casuarina-compatible strains of Frankia from Egypt. In: El-Lakany MH, Turnbull JW, Brewbaker JL, editors. Advances in Casuarina research and utilization: proceedings of the second International Casuarina Workshop, Cairo, Egypt, January 15-20, 1990 Cairo, Egypt: Desert Development Center, American University in Cairo; 1990. p. $156-64$

45. Ghodhbane-Gtari F, Nouioui I, Chair M, Boudabous A, Gtari M. 16S-23S rRNA Intergenic Spacer Region Variability in the Genus Frankia. Microb Ecol. 2010;60:487-95

46. Nouioui I, Ghodhbane-Gtari F, Montero-Calasanz MC, Göker M, MeierKolthoff JP, Schumann P, Rohde M, Goodfellow M, Fernandez MP, Normand P, Tisa LS, Klenk H-P, Gtari M. Proposal of a type strain for Frankia alni (Woronin 1866) Von Tubeuf 1895, emended description of Frankia alni, and recognition of Frankia casuarinae sp. nov. and Frankia elaeagni sp. nov. International Journal of Systematic and Evolutionary Microbiology. 2016;66: 5201-10.

47. Zhang Z, Lopez MF, Torrey JG. A comparison of cultural characteristics and infectivity of Frankia isolates from root nodules of Casuarina species. Frankia Symbioses 1984;78:79-90.

48. Normand $\mathrm{P}$, Lalonde M. Evaluation of Frankia strains isolated from provenances of two Alnus species. Can J Microbiol. 1982;28:1133-42.

49. Lalonde $\mathrm{M}$, Calvert HE, Pine $\mathrm{S}$, Calvert HE, Pine S. Isolation and use of Frankia strains in actinorhizae formation. In: Gibsen AH, editor. Current perspectives in nitrogen fixation: proceedings of the Fourth International Symposium on Nitrogen Fixation held in Canberra, Australia, 1 to $5 \mathrm{Dec}$. 1980. Amsterdam: Elsevier; 1981. p. 296-9. 
50. Hafeez F. Nitrogen fixation and nodulation in Datisca cannabina L. and Alnus nitida Endl. Islamabad, Pakistan: PhD thesis Quaid-e-Azam University; 1983.

51. Baker D, Newcomb W, Torrey JG. Characterization of an ineffective actinorhizal microsymbiont, Frankia sp. Eul1 (Actinomycetales). Can J Microbiol. 1980;26:1072-89.

52. Nouioui I, Ghodhbane-Gtari F, Montero-Calasanz MDC, Rohde M, Tisa LS, Gtari M, et al. Frankia inefficax sp. nov., an actinobacterial endophyte inducing ineffective, non nitrogen-fixing, root nodules on its actinorhizal host plants. Antonie Van Leeuwenhoek. 2016;110:313-20.

53. Tisa L, Mcbride M, Ensign JC. Studies of growth and morphology of Frankia strains EAN1 pec, Eul1 C, Cpl1, and ACN1 AG. Can J Bot. 1983;61:2768-73.

54. Smith PK, Krohn RI, Hermanson GT, Mallia AK, Gartner FH, Provenzano MD, Fujimoto EK, et al. Measurement of protein using bicinchoninic acid. Anal Biochem. 1985;150:76-85.

55. Furnholm T, Beauchemin N, Tisa LS. Development of a semi-highthroughput growth assay for the filamentous actinobacteria Frankia. Arch Microbiol. 2011;194:13-20.

56. Tisa LS, Ensign JC. Comparative physiology of nitrogenase activity and vesicle development for Frankia strains Cpl1, ACN1 ag , EAN1pec and EUN1f. Arch Microbiol. 1987;147:383-8.

57. Wang Y, Coleman-Derr D, Chen G, Gu YQ. OrthoVenn: a web server for genome wide comparison and annotation of orthologous clusters across multiple species. Nucleic Acids Res. 2015;43

58. Li L. OrthoMCL: Identification of Ortholog Groups for Eukaryotic Genomes. Genome Res. 2003;13:2178-89.

59. Enright AJ. An efficient algorithm for large-scale detection of protein families. Nucleic Acids Res. 2002;30:1575-84.

60. Lerat E, Daubin V, Ochman H, Moran NA. Evolutionary Origins of Genomic Repertoires in Bacteria. PLoS Biol. 2005;3(5):e130.

61. Goris J, Klappenbach JA, Vandamme P, Coenye T, Konstantinidis KT, Tiedje JM. DNA-DNA hybridization values and their relationship to whole-genome sequence similarities. Int J Syst Evol Microbiol. 2007;57:81-91.

62. Auch AF, Klenk H-P, Göker M. Standard operating procedure for calculating genome-to-genome distances based on high-scoring segment pairs. Stand Genomic Sci. 2010;2:142-8.

63. Lerat E, Daubin V, Moran NA. From Gene Trees to Organismal Phylogeny in Prokaryotes:The Case of the $\gamma$-Proteobacteria. PLoS Biol. 2003;1

64. Baggerly KA, Deng L, Morris JS, Aldaz CM. Differential expression in SAGE: accounting for normal between-library variation. Bioinformatics. 2003;19:1477-83.

65. Bradford M. A Rapid and Sensitive Method for the Quantitation of Microgram Quantities of Protein Utilizing the Principle of Protein-Dye Binding. Anal Biochem. 1976;72:248-54.

66. Guan S, Burlingame AL. Data Processing Algorithms for Analysis of High Resolution MSMS Spectra of Peptides with Complex Patterns of Posttranslational Modifications. Mol Cell Proteomics. 2009;9:804-10.

67. Chalkley RJ. Comprehensive Analysis of a Multidimensional Liquid Chromatography Mass Spectrometry Dataset Acquired on a Quadrupole Selecting, Quadrupole Collision Cell, Time-of-flight Mass Spectrometer: II. New Developments in Protein Prospector Allow for Reliable and Comprehensive Automatic Analysis of Large Datasets. Mol Cell Proteomics. 2005;4:1194-204.

68. Schmittgen TD, Livak KJ. Analyzing real-time PCR data by the comparative CT method. Nat Protoc. 2008;3:1101-8.

69. Kim S-K. Cloning and Characterization of the UDP Glucose/Galactose Epimerases of Oryza sativa. Journal of the Korean Society for Applied Biological Chemistry. 2009:52:315-20.

70. Jofré E, Fischer S, Príncipe A, Castro M, Ferrari W, Lagares A, et al. Mutation in a d-alanine-d-alanine ligase of Azospirillum brasilense $C d$ results in an overproduction of exopolysaccharides and a decreased tolerance to saline stress. FEMS Microbiol Lett. 2008:290:236-46.

71. Chen W, He S, Liu D, Patil GB, Zhai H, Wang F, et al. A Sweetpotato Geranylgeranyl Pyrophosphate Synthase Gene, IbGGPS, Increases Carotenoid Content and Enhances Osmotic Stress Tolerance in Arabidopsis thaliana. PLoS One. 2015;10(9):e0137623.

72. Polarek JW, Williams G, Epstein W. The products of the kdpDE operon are required for expression of the Kdp ATPase of Escherichia coli. J Bacteriol. 1992;174:2145-51.

73. Hoffmann T, Schutz A, Brosius M, Volker A, Volker U, Bremer E. High-SalinityInduced Iron Limitation in Bacillus subtilis. J Bacteriol. 2002;184:718-27.

74. Pandit A, Rai V, Sharma TR, Sharma PC, Singh NK. Differentially expressed genes in sensitive and tolerant rice varieties in response to salt-stress. J Plant Biochem Biotechnol. 2011;20:149-54.
75. Ramos JL, Martinez-Bueno M, Molina-Henares AJ, Teran W, Watanabe K, Zhang X, et al. The TetR Family of Transcriptional Repressors. Microbiol Mol Biol Rev. 2005;69:326-56.

76. Arnaouteli S, Giastas P, Andreou A, Tzanodaskalaki M, Aldridge C, Tzartos SJ, et al. Two Putative Polysaccharide Deacetylases Are Required for Osmotic Stability and Cell Shape Maintenance inBacillus anthracis. J Biol Chem. 2015;290:1346578.

77. Sévin DC, Sauer U. Ubiquinone accumulation improves osmotic-stress tolerance in Escherichia coli. Nat Chem Biol. 2014;10:266-72.

78. Youssefian S, Nakamura M, Sano H. Tobacco plants transformed with the Oacetylserine (thiol) lyase gene of wheat are resistant to toxic levels of hydrogen sulphide gas. Plant J. 1993;4:759-69.

79. Volker U, Engelmann S, Maul B, Riethdorf S, Volker A, Schmid R, et al. Analysis of the induction of general stress proteins of Bacillus subtilis. Microbiology. 1994;140:741-52

80. Kędzierska S, Staniszewska M, Węgrzyn A, Taylor A. The role of DnaK/DnaJ and GroEL/GroES systems in the removal of endogenous proteins aggregated by heat-shock from Escherichia coli cells. FEBS Lett. 1999;446:331-7.

81. Helmann JD. The extracytoplasmic function (ECF) sigma factors. Adv Microb Physiol. 2002;46:47-110.

82. Cho J-I, Lim H-M, Siddiqui ZS, Park S-H, Kim A-R, Kwon T-R, et al. Overexpression of PsGPD, a mushroom glyceraldehyde-3-phosphate dehydrogenase gene, enhances salt tolerance in rice plants. Biotechnol Lett. 2014;36:1641-8.

83. Zeng $Y$, Tan $X$, Zhang L, Long H, Wang B, Li Z, et al. A fructose-1,6biphosphate aldolase gene from Camellia oleifera: molecular characterization and impact on salt stress tolerance. Mol Breed. 2015;35:17.

84. Ventosa A, Márquez MC, Garabito MJ, Arahal DR. Moderately halophilic gram-positive bacterial diversity in hypersaline environments. Extremophiles. 1998:2:297-304.

85. Mohammad RM, Campbell WF. The search for salt-tolerant Alfalfal Rhizobium. Utah Science. 1985;46:36-7.

86. Kohler C, Lourenço RF, Bernhardt J, Albrecht D, Schüler J, Hecker M, et al. A comprehensive genomic, transcriptomic and proteomic analysis of a hyperosmotic stress sensitive a-proteobacterium. BMC Microbiol. 2015;15:71.

87. Haider S, Pal R. Integrated Analysis of Transcriptomic and Proteomic Data. Current Genomics. 2013;14:91-110.

88. Rushton PJ, Somssich IE. Transcriptional control of plant genes responsive to pathogens. Curr Opin Plant Biol. 1998;1:311-5.

89. Humpel A, Gebhard S, Cook GM, Berney M. The SigF Regulon in Mycobacterium smegmatis Reveals Roles in Adaptation to Stationary Phase, Heat, and Oxidative Stress. J Bacteriol. 2010;192:2491-502.

90. Helmann JD. Anti-sigma factors. Curr Opin Microbiol. 1999;2:135-41.

91. Paget MSB, Molle V, Cohen G, Aharonowitz Y, Buttner MJ. Defining the disulphide stress response in Streptomyces coelicolor A3(2): identification of the sigmaR regulon. Mol Microbiol. 2001;42:1007-20.

92. Hughes KT, Mathee K. The Anti-Sigma Factors. Annu Rev Microbiol. 1998;52: 231-86.

93. Kidric M, Kos J, Sabotic J. Proteases and their endogenous inhibitors in the plant response to abiotic stress. Botanica Serbica. 2014;38:139-58.

94. López C, Heras H, Garda H, Ruzal S, Sánchez-Rivas C, Rivas E. Biochemical and biophysical studies of Bacillus subtilis envelopes under hyperosmotic stress. Int J Food Microbiol. 2000;55:137-42.

95. Piuri M, Sanchez-Rivas C, Ruzal S. Cell wall modifications during osmotic stress in Lactobacillus casei. J Appl Microbiol. 2005;98:84-95.

96. Miller KJ, Wood JM. Osmoadaptation By Rhizosphere Bacteria. Annu Rev Microbiol. 1996;50:101-36.

97. Prã-ncipe A, Jofrãc E, Alvarez F, Mori G. Role of a serine-type d-alanyl-dalanine carboxypeptidase on the survival of Ochrobactrum sp. 11a under ionic and hyperosmotic stress. FEMS Microbiol Lett. 2009;295:261-73.

98. Klein W, Weber MHW, Marahiel MA. Cold shock response of Bacillus subtilis: isoleucine-dependent switch in the fatty acid branching pattern for membrane adaptation to low temperatures. Journal of Bacteriology. 1999;181:5341-5349.

99. Beck HC, Hansen AM, Lauritsen FR. Catabolism of leucine to branched-chain fatty acids in Staphylococcus xylosus. J Appl Microbiol. 2004;96:1185-93.

100. Wu D, Cai S, Chen M, Ye L, Chen Z, Zhang H, et al. Tissue Metabolic Responses to Salt Stress in Wild and Cultivated Barley. PLoS One. 2013;8(1):e55431.

101. Morita YS, Yamaryo-Botte Y, Miyanagi K, Callaghan JM, Patterson JH, Crellin PK, et al. Stress-induced Synthesis of Phosphatidylinositol 3-Phosphate in Mycobacteria. J Biol Chem. 2010;285:16643-50. 